\title{
Renegotiating situativity: transformations of local herbal knowledge in a Western Alpine valley during the past 40 years
}

\author{
Michele F. Fontefrancesco ${ }^{1}$ and Andrea Pieroni ${ }^{12^{*}}$ (D)
}

\begin{abstract}
Background: Mountain environments are fragile socio-ecological systems and the conservation of their biological and cultural diversities - seen as co-evolving, strongly intertwined entities — represents a crucial issue for fostering their sustainability. Very few ethnobiological studies have assessed in the mountainous regions of Europe how local botanical knowledge, which represents a vital portion of the local environmental knowledge (LEK), changes over time, although this may be quintessential for a better understanding of the factors influencing how knowledge and practices are shaped, eroded, or even re-created.
\end{abstract}

Methods: In the current study, we compared the gathering and use of local medicinal plants in the Upper Sangone Valley, Western Italian Alps, Piedmont (NW Italy) as described in a field study conducted in the midseventies and published in 1977 and those arising from field research that we conducted in the spring of 2015 and 2018, during which time ethnobotanical and ethnomycological information concerning both folk medicinal and wild food uses was obtained via 47 in-depth open and semi-structured interviews with community members.

Results: In total, one hundred thirty folk taxa represent the past and present medicinal and wild food plant/ mushroom heritage of the Sangone Valley: 26 herbal taxa were recorded 40 years ago only; 68 herbal and wild food taxa have been recorded in the current study only; and 36 herbal taxa have been continuously used during the last 40 years. There were no remarkable quantitative differences between the two diachronic medicoethnobotanical datasets, but the qualitative differences were substantial. The gathering and use of some medicinal plants growing in meadows, forests and higher mountain environments (i.e. Arctostaphylos, Filipendula, Hepatica, Larix, Laserptium, Picea, Polygonatum, Primula, Tussilago and Veronica spp.) disappeared, whereas the collection of plant genera growing in more anthropogenic environments or possibly promoted via popular books and media has been newly introduced (i.e. Aloysia, Apium, Brassica, Crataegus, Epilobium, Fumaria, Geranium, Juniperus, Melissa, Rubus, Rumex, Sedum, Silybum, Taraxacum and Vaccinium spp.).

\footnotetext{
* Correspondence: a.pieroni@unisg.it

'University of Gastronomic Sciences, Piazza Vittorio Emanuele 9, 12060 Bra,

Pollenzo, Italy

${ }^{2}$ Department of Medical Analysis, Tishk International University, Qazi

Muhammad, Erbil, Kurdistan 44001, Iraq
}

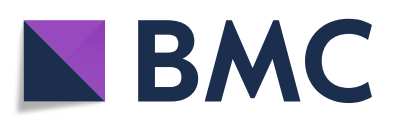

(c) The Author(s). 2020 Open Access This article is licensed under a Creative Commons Attribution 4.0 International License, which permits use, sharing, adaptation, distribution and reproduction in any medium or format, as long as you give appropriate credit to the original author(s) and the source, provide a link to the Creative Commons licence, and indicate if changes were made. The images or other third party material in this article are included in the article's Creative Commons licence, unless indicated otherwise in a credit line to the material. If material is not included in the article's Creative Commons licence and your intended use is not permitted by statutory regulation or exceeds the permitted use, you will need to obtain permission directly from the copyright holder. To view a copy of this licence, visit http://creativecommons.org/licenses/by/4.0/ The Creative Commons Public Domain Dedication waiver (http://creativecommons.org/publicdomain/zero/1.0/) applies to the data made available in this article, unless otherwise stated in a credit line to the data. 


\begin{abstract}
(Continued from previous page)
Conclusion: The findings show a renegotiation of the situativity that for centuries forged the embeddedness of local communities in their natural environments, probably heavily informed in the past by prevalent pastoralist and forest-centred activities and thus by a deeper knowledge of higher mountain and forest environments. The rearrangement of a more domestic and more "globalized" herbal knowledge system was possibly inspired by new urban residents, who started to populate the valley at the end of the Seventies, when the original inhabitants abandoned their homes for the urban centres of the Piedmontese plain. The current study suggests that future directions of ethnobiological research should more carefully look at the adaptive capacity of LEK systems.
\end{abstract}

Keywords: Ethnobotany, Alps, LEK, Social change, Mountain development

\section{Introduction}

In the past decades, local environmental knowledge (LEK) of mountain communities has increasingly been at the centre of important field researches and public debates as it is nowadays widely considered a precious resource for sustainable development [1-6]. The value of LEK, however, has often been perceived in opposition to modernity which draws from the peripherality of the communities and their distance from cities, the main perceived catalyst centres of "modernity". This assumption, though, has reiterated a long-standing dichotomic model that represents part of the problematic scientific debate which views the LEK of marginal communities in opposition to modernity $[7,8]$. The relationship between LEK and modernity has often been described with binary conceptualizations, such as the science of the concrete vs. "the sciences" [9], tacit knowledge vs. scientific knowledge [10], oral knowledge vs. written knowledge [11], Indigenous Knowledge vs. Western knowledge [12] and Traditional Knowledge vs. modern knowledge [13].

"Situated" local ecological knowledge (LEK) refers to a system of knowledge, practices and beliefs that is profoundly embedded within a given socio-ecological endroit [14]. Lauer and Aswani [15] described how LEK in the Western Salomon Islands overcame the distinction between cognitive aspects and other modalities of knowing and how it is deeply intertwined with everyday ecological practices. Ecological knowing and practicing is more than a mere, even complex, system and Whyte [16] argued that this LEK is actually to be seen as a collaborative concept and that scientists, environmentalists and local communities should try to create long-term processes that allow the different implications of approaches to knowledge in relation to stewardship goals to be responsibly considered. In this perspective, the concept of situated LEK has become a useful tool in regional planning linked, in particular, with rural development [17]. More broadly, the debate about situated knowledge, however, highlights the dynamicity of local knowledge and examines the possible drivers of change in order to support local communities and environmental preservation.

In a recent review that assessed the global literature and analysed the drivers of various types of LEK transformations [18], the authors postulated that the impoverishment of LEK may be driven by globalization, modernization, and market integration. Moreover, they highlighted the unsymmetrical loss of LEK, with losses being more markedly recorded in medicinal and botanical domains.

Changes in LEK measured as knowledge and practices concerning the ecosystem have been the subject of a few studies in recent years $[19,20]$. Liu et al. [19] described the industry-, government- and culture-driven changes of LEK of Tao fly fishing culture in Taiwan and found that various external factors have contributed to LEK changes, such as inappropriate government policies, modern science and technology, education and the market economy. Duenn et al. [20] studied the perception of invasive alien species among Rabari pastoralists from Gujarat, India, and the results showed that alien species invasion was not perceived as a major problem; the authors postulated that the adaptive capacity of LEK systems and a slow rate of environmental change directly attributable to alien species may explain this apparent "paradox".

However, changes in folk botanical knowledge, assessed using comparable research methods (ethnography-based techniques, e.g. face-to-face interviews), remain poorly investigated. Some diachronic comparisons in ethnobotany have been attempted, with interesting results, in Eastern Europe [21-26] and, more recently, in Southern Europe [27], while beyond Europe this has been more sporadically done [28, 29]. In particular, in Poland, a comprehensive historical study showed that LEK linked to the gathering of wild green vegetables has gradually decreased, mainly due to replacement by a few cultivated vegetables; this process started in the nineteenth century, but became more pronounced during the twentieth century [22]. Moreover, a review focusing on wild food plant gathering in Europe [30] has underlined not only the decline of "traditional" foraging but also the remarkable emergence of a new, "recreational" foraging, practiced by urban classes, often popularized by trained botanists, organic farmers, chefs, and food activists, and whose real impact among the majority of the population in Western societies has still to be properly assessed. Schunko et al. [31] well 
demonstrated a decade ago that the motivation of the resurgent popularity of wild plant gathering among organic farmers in Austria is to be attributed to an "internalization" of motivations, i.e. the pleasure of the activity itself, while diverse authors in Europe ([32], and chapters therein [33]) discussed how the resilience or resurgence of foraging may be linked to the increasing attention communities and citizens pay to their local/regional cultural identities and to the perceived healthy properties of the wild food plants.

On the herbal ethnobotanical side, changes in medicinal plant use in Estonia between 1888 and 1994 showed that the use of garlic, hose chestnuts and mint increased, whereas the use of barley, orchids, Paris quadrifolia and Briza media significantly decreased [23], thus supporting the idea that during the past century the utilization of plants depending on human influence was remarkably amplified.

On the other hand, the lack of extensive literature providing diachronic ethnobotanical data is due to the fact that the few field studies carried out decades ago were often conducted by researchers (primarily folklorists or local historians) who were not able to present reliable botanical identifications or, more rarely, by biologists who had never been exposed to the social sciences. We believe that these limitations can greatly affect the validity of comparative works much more than is generally assumed by many researchers. However, in one study that was conducted in the mid-seventies (most likely in 1976) in the Upper Sangone Valley, a tiny valley of the Western Italian Alps [34], researchers documented folk herbal practices via face-to-face interviews with locals. We therefore decided to conduct the current field research in exactly the same area, in order to analyse the ethnobotanical picture 40 years later.

The present paper provides a contribution to the aforementioned debate by exploring the changes in LEK in a Western Alpine valley during the past 40 years, using as a proxy a comparison between the medicinal plants gathered and used by the local community in the mid-seventies and those gathered and used in the years 2015-2018.

The specific objectives of this study therefore were:

(a) to investigate the current ethnobotanical heritage (including both wild food and herbal plant and mushroom uses) of the Upper Sangone Valley;

(b) to compare the recorded herbal uses with those recorded forty years earlier;

(c) to interpret possible differences in both ecological and/or cultural terms and to discuss the diachronic transformation of local plant knowledge.

\section{Materials and methods}

\section{Selection of the ethnobotanical literature}

A decade ago, our research group started an investigation on the historical ethnobotanical literature of Italy from 1884 to the 1980s [35, 36] and selected those works which included both reliable botanical identifications (i.e. works indicating voucher specimens, or studies conducted by academic botanists or, even better, botanical taxonomists) and a clear description that the ethnobotanical information was collected via face-to-face interviews. At the end of this months-long work, we obtained no more than a dozen field studies conducted between 1955 and 1980 in NW, NE and Central Italy, which could be considered appropriate for diachronic comparison. Among this restricted group, one study conducted in the mid-seventies in the Western Alps emerged [34]. This particular field study was conducted in the Upper Sangone Valley and subsequently published in an Italian hospital pharmacy journal in 1977. The work was conducted, without the collection of vouchers, by academic botanists via face-to-face interviews with locals, focusing specifically on local wild and cultivated medicinal plants gathered and used in domestic herbal medicine or as a medicinal food (i.e. herbal liqueurs and other food preparations consumed with the express purpose of treating certain illnesses). The work did not document, however, any frequency of quotation, or frequency of use, of the cited medicinal plants, or details regarding the areas in which the plants were collected.

\section{Current field study}

The current field study was conducted in the same area where the aforementioned study was conducted in the Seventies: the Upper Sangone Valley, Cottian Alps, Piedmont, NW Italy (Fig. 1). The following small villages were visited: Alpe Palè $\left(\mathrm{N} 45^{\circ} 03^{\prime} 56^{\prime \prime}\right.$ E $7^{\circ} 13^{\prime} 32^{\prime \prime}$, Coazze Muncipality, $1356 \mathrm{~m}$ a.s.l.; approximately 10 inhabitants); Borgata Tora (N 45 00' 33" E $7^{\circ} 18^{\prime} 11^{\prime \prime}$, Giaveno Municipality, $994 \mathrm{~m}$ a.s.l.; approximately 20 inhabitants); Cervelli (N 45 03' 13" E $7^{\circ} 16^{\prime} 06^{\prime \prime}$, Coazze Municipality, $881 \mathrm{~m}$ a.s.l., approximately 40 inhabitants); Colle Braida (N 45 04' 59" E $7^{\circ} 19^{\prime} 59^{\prime \prime}$, Valgioie Municipality, 1008 $\mathrm{m}$ a.s.l.; 10 inhabitants); Mollar dei Franchi (N 45 01' 28" E $7^{\circ} 20^{\prime} 17^{\prime \prime}$, Giaveno Municipality, $563 \mathrm{~m}$ a.s.l.; 50 inhabitants); Roccette (N 45 01' 37" E $7^{\circ} 17^{\prime} 45^{\prime \prime}$, Giaveno Municipality, $780 \mathrm{~m}$ a.s.l.; 60 inhabitants); and Prè Fieul (N 45 01' 42" E 7 $16^{\prime} 15^{\prime \prime}$, Giaveno Municipality, 1263 $\mathrm{m}$ a.s.l.; approximately 20 inhabitants). The main centre of Coazze (N 45 03' 07" E $7^{\circ} 18^{\prime} 02^{\prime \prime}, 750$ m a.s.l., approximately 2700 inhabitants) was also included, since a few inhabitants maintain links to pastures in higher valleys.

The field study was carried out by the two authors with the help of some trained students (see acknowledgments) in the spring of 2015 and 2018. In-depth open and semi-structured interviews were conducted with community members $(n=47$, average age $65 ; 21$ interviews were conducted in 2015 and 26 in 2018) in the aforementioned eight settlements. Participants were 


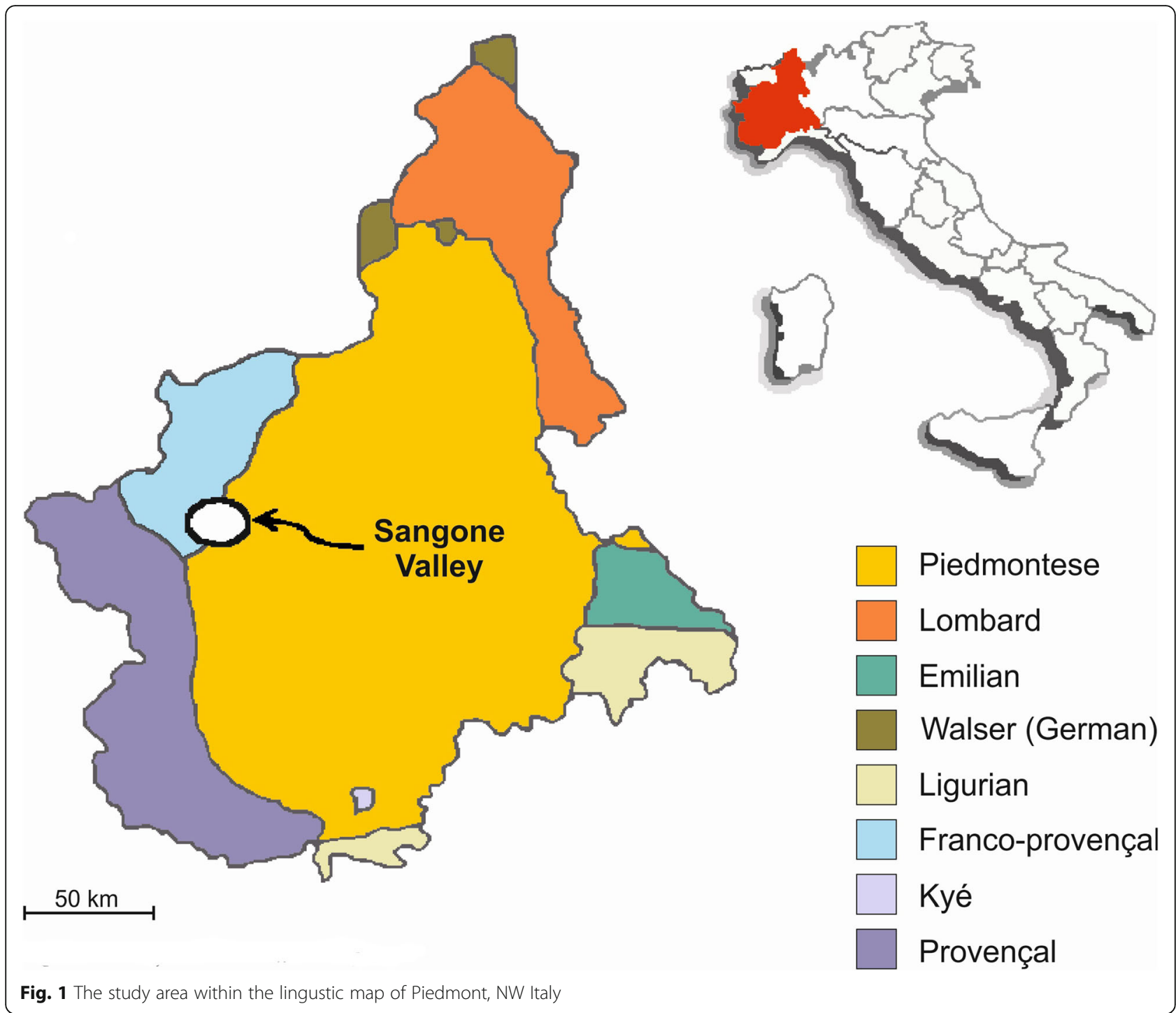

selected using snowball sampling techniques, focusing specifically on those elderly community-members (e.g. farmers, housewives) who still have connections to an agro-pastoral way of life. Informants were asked about current and recent past gathering and use of (a) local wild food plants; (b) wild and domesticated medicinal and veterinary plants; and (c) mushrooms for food and/ or medicine. The second category (b) included both proper medicinal plants and also plant-based medicinal foods (i.e. herbal liqueurs and other food preparations consumed with the express purpose of treating certain illnesses).

Specifically, study participants were asked about the local plant name(s) and plant part(s) used, as well as specific details about their manipulation/preparation and actual herbal or food use(s). Interviews were conducted in Italian. Prior informed consent was always verbally obtained prior to the interviews and researchers adhered to the ethical guidelines of the International Society of Ethnobiology [37]. During the interviews, informants were always asked to show the quoted plants or, at least, to describe them. Botanical excursions both around the house and in the mountains were additionally conducted in order to collect botanical specimens, which are deposited at the Herbarium of the University of Gastronomic Sciences. Taxonomic identifications were carried out by the first author according to the Flora of Italy [38], while botanical nomenclature was later standardized using The Plant List database [39]. Family assignments follow the Angiosperm Phylogeny Group IV system [40] and mushroom nomenclature follows the Index Fungorum database [41].

\section{Study area}

The surrounding landscape is typical of pre-Alpine and Alpine habitats. Chestnut and beech tree forests dominate 
up to $1000 \mathrm{~m}$, while higher elevations are covered by fir, pine and birch forests, as well as grasslands with rhododendron and blueberry bushes at over $2500 \mathrm{~m}$ a.s.l. The upper part of the territory is occupied by OrsieraRocciavrè Natural Park, a protected area of approximately 10,000 ha, established by the region of Piedmont in 1980 . The park extends into the Northern Cottian Alps, the boundaries of which run, on average, at an altitude of $1400 \mathrm{~m}$ a.s.l., and the most important mountain peaks included within the park reach nearly $3000 \mathrm{~m}$ a.s.l. The altitude of thermal zero in winter is $1700 \mathrm{~m}$ a.s.l. The territory is characterized by relatively low rainfall, which is among the lowest in NW Italy. On average, maximum rainfall is in May and October and the minimum is in January and July. The geomorphological characteristics of the park territory allow many animal species to find suitable habitat, among them hares, rock ptarmigans, marmots, chamois and mouflons.

As in other valleys in the Western Alps [42-45], the traditional economy of the communities in the Sangone Valley relied on a multifunctional agricultural model based on small family farms, which combined the cultivation of cereals (mainly barley, rye, and maize) with that of vegetables (such as cabbages, carrots, onions, potatoes, onions, turnips) and fruits (apples, pears). In addition, and more importantly, it also included cattle, sheep and goat pastoralism, which supported local dairy production, while hunting and gathering practices (which included the collection of chestnuts, mushrooms and herbs used for both food and medicine) were mainly carried out in forests and higher pastures. This model was still followed in the early decades of the twentieth century [46]. Despite the importance of agriculture, the manufacturing industry has played a key role in the valley since the late nineteenth century. The Sangone Valley is located on one of the main transit routes between Italy and France [47], and its municipalities are easily accessible from Turin, one of the most important industrial cities in Italy. This allowed rapid development of the manufacturing industry in the valley beginning in the nineteenth century [48-50] and fostered the emergence of a mixed household economy that linked wage-earning to multifunctional farming activities. Moreover, at the beginning of the twentieth century, the valley became one of the first destinations for Alpine tourism [51], primary associated with trekking and cross-country skiing, which further complexified the local economy. As in the rest of the Alpine region [52], the post-WWII period marked the decline of agriculture as an economic sector of the valley, with the reduction of cultivated land in the valley [53], as well as specialization of agricultural production (e.g. apples, pears). While in the 1960s and 1970s, the valley experienced fast industrial development, in the following decades, like the rest of the region [54], the economy of the Sangone Valley opened to the service sector, accelerating the agricultural decline. In this transformation, tourism has played a vital role, expanding its reach and encompassing initiatives linked to the promotion of local cultural and gastronomic heritage [55, 56].

The local language of the Upper Sangone Valley, referred to by locals as patois, is Franco-Provençal (also known as Francoprovençal, Arpitan or Romand), which is mainly used only in the domestic arena by elderly community members; both standard Italian and the Piedmontese dialect are largely dominant among the middle and younger generations and in all communication with external visitors. Franco-Provençal represents a dialect group within the Gallo-Romance language family, which is also spoken in East and Central France, Western Switzerland, NW Italy, and in two tiny enclaves in SE Italy. Its current use within the domestic arena is decreasing, although some organizations are attempting to preserve this linguistic heritage through cultural events, educational platforms and some publishing activities [57].

\section{Data analysis}

The medicinal botanical data recorded in 2015 and 2018 were tabulated and compared with those arising from the medical-ethnobotanical fieldwork that was conducted in the mid-seventies in the same area [34] via a matrix describing the plant genera traditionally gathered and used for medicinal purposes nowadays and/or 40 years ago, which was then visualised in a Venn diagram.

Additionally, the recorded plant reports were qualitatively compared with the most comprehensive worldwide wild food plant compendia [58, 59], the Italian medical ethnobotanical database [60], an ethnobotanical survey carried out in a nearby valley one decade ago [61], and a few recent, sporadic ethnobotanical studies conducted on the Italian side of the Alps [62-65], in order to point out possible novel reports (i.e. reports not previously recorded in the Italian ethnobotanical literature).

Moreover, the most important written popular sources of herbal knowledge published in Italy from the 1970s until now were also considered [5, 66-72], in order to qualitatively assess the possible correspondence between "new" recorded herbal customs and their recurrent presence in this literature.

\section{Results \\ Plants and mushrooms for food and medicine in the Sangone Valley}

In total, one hundred thirty folk taxa (i.e. taxa linked to a single folk plant/mushroom name) were recorded, representing the past and present medicinal and food heritage of the Sangone Valley (Table 1):

- 26 taxa used as medicine were recorded 40 years ago only; 
Table 1 Local wild food and medicinal plants gathered and used in the study area

\begin{tabular}{|c|c|c|c|c|c|}
\hline $\begin{array}{l}\text { Gathered botanical taxon/ } \\
\text { taxa, family/-ies and } \\
\text { voucher specimen code(s) }\end{array}$ & $\begin{array}{l}\text { Local plant } \\
\text { name(s) }\end{array}$ & Used parts & $\begin{array}{l}\text { Folk use recorded in } 1976 \\
\text { (preparation) }\end{array}$ & $\begin{array}{l}\text { Folk use recorded in the } \\
\text { current field study } \\
\text { (preparation) }\end{array}$ & $\begin{array}{l}\text { Frequency of use in the } \\
\text { current field study }\end{array}$ \\
\hline $\begin{array}{l}\text { Acer pseudoplatanus L., } \\
\text { Aceraceae, } \\
\text { UNISGSANG18001 }\end{array}$ & Piè & Leaves & & $\begin{array}{l}\text { FOO: wrapping for butter; } \\
\text { VET: fodder for goats }\end{array}$ & $\begin{array}{l}\text { N/A (remembered from } \\
\text { the past) }\end{array}$ \\
\hline $\begin{array}{l}\text { Achillea herba-rotta All., A. } \\
\text { millefolium L., and } \\
\text { possibly A. nana L., } \\
\text { Asteraceae, } \\
\text { UNISGSANG15001 (A. } \\
\text { herba-rotta) and } \\
\text { UNISGSANG18005 (A. } \\
\text { millefolium) }\end{array}$ & Marmin & Whole plant & $\begin{array}{l}\text { HER: diuretic, febrifuge } \\
\text { (tea) }\end{array}$ & $\begin{array}{l}\text { FOO/HER: liqueurs } \\
\text { (considered diuretic); HER: } \\
\text { diuretic (tea); anti- } \\
\text { hematomas and anti- } \\
\text { bruises (poultice with lard } \\
\text { and vinegar) }\end{array}$ & Very common \\
\hline $\begin{array}{l}\text { Adianthum hispidulum Sw., } \\
\text { Pteridaceae, } \\
\text { UNISGSANG18045 }\end{array}$ & Fugèt & Aerial parts & & $\begin{array}{l}\text { HER: enhancing blood } \\
\text { circulation, emmenagogue } \\
\text { (tea) }\end{array}$ & Rare \\
\hline Alcea rosea L., Malvaceae & & & $\begin{array}{l}\text { HER: anti-kidney stones } \\
\text { (decoction) }\end{array}$ & & \\
\hline $\begin{array}{l}\text { Alchemilla } \\
\text { xanthochlora Rothm., } \\
\text { Rosaceae }\end{array}$ & Cuculin & Leaves & & $\begin{array}{l}\text { HER: anti-dysmenorrhea } \\
\text { (external washes) }\end{array}$ & Rare \\
\hline $\begin{array}{l}\text { Allium cepa } \mathrm{L} ., \\
\text { Amaryllidaceae }\end{array}$ & Siola & Bulbs & & $\begin{array}{l}\text { HER: bechic (tea), digestive } \\
\text { (broth), anti-inflammatory } \\
\text { and panacea (cooked) }\end{array}$ & Very common \\
\hline $\begin{array}{l}\text { Allium sativum L., } \\
\text { Amaryllidaceae }\end{array}$ & $\mathrm{Ai}$ & Bulbs & $\begin{array}{l}\text { HER: vermifuge (also in } \\
\text { necklaces to be worn } \\
\text { during the night) }\end{array}$ & $\begin{array}{l}\text { HER: hypotensive (food); } \\
\text { vermifuge in children (slice } \\
\text { of bread rubbed with } \\
\text { garlic) }\end{array}$ & Fairly common \\
\hline $\begin{array}{l}\text { Allium schoenoprasum L., } \\
\text { Amaryllidaceae }\end{array}$ & Erba cipollina & Leaves & & FOO: seasoning & Rare \\
\hline $\begin{array}{l}\text { Allium ursinum L., } \\
\text { Amaryllidaceae }\end{array}$ & Aiet & Leaves & & FOO: salads, soups & Rare \\
\hline $\begin{array}{l}\text { Alnus incana (L.) Moench, } \\
\text { Betulaceae, } \\
\text { UNISGSANG15002 }\end{array}$ & Verna & Leaves & & $\begin{array}{l}\text { HER: anti-back pain (com- } \\
\text { press of dried leaves) }\end{array}$ & Rare \\
\hline $\begin{array}{l}\text { Aloysia citrodora Palau, } \\
\text { Verbenaceae, } \\
\text { UNISGSANG18057 }\end{array}$ & Limonaria & Leaves & & HER: digestive (tea) & Rare \\
\hline $\begin{array}{l}\text { Althaea officinalis L., } \\
\text { Malvaceae, } \\
\text { UNISGSANG15003 }\end{array}$ & Redaui & Roots & $\begin{array}{l}\text { HER: anti-kidney stones } \\
\text { (decoction) }\end{array}$ & $\begin{array}{l}\text { HER: anti-toothache in chil- } \\
\text { dren (tea made from fresh } \\
\text { roots) }\end{array}$ & Rare \\
\hline $\begin{array}{l}\text { Angelica archangelica L. } \\
\text { and A. sylvestris L., } \\
\text { Apiaceae }\end{array}$ & Angelica & $\begin{array}{l}\text { Roots and } \\
\text { aerial parts }\end{array}$ & $\begin{array}{l}\text { HER: digestive (liqueur } \\
\text { made from the roots) }\end{array}$ & $\begin{array}{l}\text { HER: anti-carbuncles (com- } \\
\text { press of aerial parts) }\end{array}$ & Rare \\
\hline $\begin{array}{l}\text { Apium graveolens L., } \\
\text { Apiaceae }\end{array}$ & Serli & Leaves & & $\begin{array}{l}\text { HER: male aphrodisiac } \\
\text { (consumed) }\end{array}$ & Rare \\
\hline $\begin{array}{l}\text { Arctium lappa L., } \\
\text { Asteraceae, } \\
\text { UNISGSANG15004 }\end{array}$ & Cicapuoi & Leaves & HER: bechic (decoction) & $\begin{array}{l}\text { HER: hepato-protector } \\
\text { (decoction) }\end{array}$ & Rare \\
\hline $\begin{array}{l}\text { Arctostaphylos uva-ursi (L.) } \\
\text { Spreng., Ericaceae }\end{array}$ & & Leaves & $\begin{array}{l}\text { HER: anti-urinary tract in- } \\
\text { fections (decoction) }\end{array}$ & & \\
\hline $\begin{array}{l}\text { Armillaria mellea (Vahl.) } \\
\text { P.Kumm., Physalacriaceae }\end{array}$ & Famiole & Fruiting body & & FOO: pickled in oil & Very common \\
\hline $\begin{array}{l}\text { Arnica montana L., } \\
\text { Asteraceae, } \\
\text { UNISGSANG18006, } \\
\text { UNISGSANG15005 }\end{array}$ & Arnica, Bertolica & Flowers & & $\begin{array}{l}\text { HER: for treating many skin } \\
\text { inflammations, anti-bruises, } \\
\text { anti-rheumatic (oleolite or } \\
\text { alcoholic macerate, exter- } \\
\text { nally applied); anti-flu } \\
\text { (inhaled) }\end{array}$ & Very common \\
\hline
\end{tabular}


Table 1 Local wild food and medicinal plants gathered and used in the study area (Continued)

\begin{tabular}{|c|c|c|c|c|c|}
\hline $\begin{array}{l}\text { Gathered botanical taxon/ } \\
\text { taxa, family/-ies and } \\
\text { voucher specimen code(s) }\end{array}$ & $\begin{array}{l}\text { Local plant } \\
\text { name(s) }\end{array}$ & Used parts & $\begin{array}{l}\text { Folk use recorded in } 1976 \\
\text { (preparation) }\end{array}$ & $\begin{array}{l}\text { Folk use recorded in the } \\
\text { current field study } \\
\text { (preparation) }\end{array}$ & $\begin{array}{l}\text { Frequency of use in the } \\
\text { current field study }\end{array}$ \\
\hline $\begin{array}{l}\text { Artemisa absinthium L., } \\
\text { Asteraceae, } \\
\text { UNISGSANG15007 }\end{array}$ & $\begin{array}{l}\text { Erba banci, } \\
\text { Incens }\end{array}$ & $\begin{array}{l}\text { Leaves and } \\
\text { flowers }\end{array}$ & HER: vermifuge (decoction) & $\begin{array}{l}\text { VET: enhancing placenta } \\
\text { expulsion (fodder in cows) }\end{array}$ & Rare \\
\hline $\begin{array}{l}\text { Artemisia genipi Weber ex } \\
\text { Stechm. and possibly A. } \\
\text { glacialis L. and A. } \\
\text { umbelliformis Lam., } \\
\text { Asteraceae, } \\
\text { UNISGSANG15006 (A. } \\
\text { genipi) }\end{array}$ & $\begin{array}{l}\text { Genepì ( } A \text {. } \\
\text { genipi), Genepì } \\
\text { fumela (A. } \\
\text { glacialis and } \\
\text { umbelliformis) }\end{array}$ & Aerial parts & HER: digestive (tea) & $\begin{array}{l}\text { FOO/HER: liqueurs } \\
\text { (considered digestive); } \\
\text { HER: digestive (tea) }\end{array}$ & Fairly common \\
\hline $\begin{array}{l}\text { Aruncus dioicus (Walter) } \\
\text { Fernald., Rosaceae }\end{array}$ & Spars servai & Shoots & & FOO: boiled & Fairly common \\
\hline $\begin{array}{l}\text { Betula pendula Roth, } \\
\text { Betulaceae, } \\
\text { UNISGSANG18010 }\end{array}$ & Bès & Sap & & $\begin{array}{l}\text { HER: blood depurative } \\
\text { (drunk) }\end{array}$ & Rare \\
\hline $\begin{array}{l}\text { Boletus aureus Schaeff., } \\
\text { Boletaceae }\end{array}$ & Moru & Fruiting body & & $\begin{array}{l}\text { FOO: salads, sautéed, fried, } \\
\text { pickled }\end{array}$ & Very common \\
\hline $\begin{array}{l}\text { Boletus edulis Bull., } \\
\text { Boletaceae }\end{array}$ & Bouloi & Fruiting body & & $\begin{array}{l}\text { FOO: salads, sautéed, fried, } \\
\text { pickled }\end{array}$ & Very common \\
\hline $\begin{array}{l}\text { Brassica oleracea L. var. } \\
\text { sabauda, Brassicaceae }\end{array}$ & Coi & Leaves & & $\begin{array}{l}\text { HER: anti-bruises (topically } \\
\text { applied) }\end{array}$ & Rare \\
\hline $\begin{array}{l}\text { Brassica rapa } \mathrm{L} ., \\
\text { Brassicaceae }\end{array}$ & Rava & Roots & $\begin{array}{l}\text { HER: bechic (also for } \\
\text { whooping cough; slices of } \\
\text { the root mixed with honey } \\
\text { and left to macerate } 3 \\
\text { days) }\end{array}$ & $\begin{array}{l}\text { FOO: external "peel" dried } \\
\text { and cooked in milk when } \\
\text { vegetables were scarce in } \\
\text { the spring }\end{array}$ & $\begin{array}{l}\text { N/A (remembered from } \\
\text { the past) }\end{array}$ \\
\hline $\begin{array}{l}\text { Calluna vulgaris (L.) Hull, } \\
\text { Ericaceae, } \\
\text { UNISGSANG15009 }\end{array}$ & Bruri & Leaves & & $\begin{array}{l}\text { HER: anti-diarrhoeic (de- } \\
\text { coction); VET: fodder for } \\
\text { rabbits }\end{array}$ & Rare \\
\hline $\begin{array}{l}\text { Carlina acanthifolia All., } \\
\text { Asteraceae, } \\
\text { UNISGSANG18007 }\end{array}$ & Cardun, Pugnu & $\begin{array}{l}\text { Flowers and } \\
\text { flower } \\
\text { receptacles }\end{array}$ & & $\begin{array}{l}\text { FOO: snack (flower } \\
\text { receptacles); FOO/HER: } \\
\text { digestive (liqueur made } \\
\text { from the flowers) }\end{array}$ & $\begin{array}{l}\text { Fairly common (FOO); } \\
\text { rare (FOO/HER) }\end{array}$ \\
\hline $\begin{array}{l}\text { Carum carvi L., Apiaceae, } \\
\text { UNISGSANG15010 }\end{array}$ & $\begin{array}{l}\text { Cumino, } \\
\text { Cümmel }\end{array}$ & Fruits & & $\begin{array}{l}\text { FOO/HER: liqueur } \\
\text { (considered digestive) }\end{array}$ & Rare \\
\hline $\begin{array}{l}\text { Castanea sativa Mill., } \\
\text { Fagaceae, } \\
\text { UNISGSANG18022 }\end{array}$ & Castagn & Fruits & & $\begin{array}{l}\text { FOO: soups with milk and } \\
\text { potatoes, roasted, jams, } \\
\text { sweets, liqueurs }\end{array}$ & Very common \\
\hline $\begin{array}{l}\text { Ceterach officinarum Willd., } \\
\text { Aspleniaceae }\end{array}$ & & Aerial parts & $\begin{array}{l}\text { HER: diuretic, vermifuge } \\
\text { (decoction, taste } \\
\text { sometimes improved with } \\
\text { a few mint leaves) }\end{array}$ & & \\
\hline $\begin{array}{l}\text { Cetraria islandica (L.) Ach., } \\
\text { Parmeliaceae, } \\
\text { UNISGSANG15011 }\end{array}$ & Lica & $\begin{array}{l}\text { Thallus } \\
\text { (traditionally } \\
\text { gathered in } \\
\text { fields over 2, } \\
000 \mathrm{~m} \text { a.s.l. on } \\
\text { the 2nd } \\
\text { Sunday of July) }\end{array}$ & $\begin{array}{l}\text { HER: bechic and } \\
\text { demulcent (decoction, } \\
\text { sometimes together with } \\
\text { lime tree flowers) }\end{array}$ & $\begin{array}{l}\text { HER: bechic and anti- } \\
\text { inflammatory of the re- } \\
\text { spiratory tract (decoction } \\
\text { or syrup) }\end{array}$ & Fairly common \\
\hline $\begin{array}{l}\text { Chantarellus cibarius Fr., } \\
\text { Cantharellaceae }\end{array}$ & Garitula & Fruiting body & & FOO: soups, sautéed, fried & Very common \\
\hline $\begin{array}{l}\text { Chelidonius majus L., } \\
\text { Papaveraceae }\end{array}$ & Erba di poret & Latex & $\begin{array}{l}\text { HER: anti-warts (topically } \\
\text { applied) }\end{array}$ & & \\
\hline $\begin{array}{l}\text { Chenopodium bonus- } \\
\text { henricus L., Amaranthaceae, }\end{array}$ & Spinas sarvai & Leaves & & FOO: cooked & Fairly common \\
\hline
\end{tabular}


Table 1 Local wild food and medicinal plants gathered and used in the study area (Continued)

\begin{tabular}{|c|c|c|c|c|c|}
\hline $\begin{array}{l}\text { Gathered botanical taxon/ } \\
\text { taxa, family/-ies and } \\
\text { voucher specimen code(s) }\end{array}$ & $\begin{array}{l}\text { Local plant } \\
\text { name(s) }\end{array}$ & Used parts & $\begin{array}{l}\text { Folk use recorded in } 1976 \\
\text { (preparation) }\end{array}$ & $\begin{array}{l}\text { Folk use recorded in the } \\
\text { current field study } \\
\text { (preparation) }\end{array}$ & $\begin{array}{l}\text { Frequency of use in the } \\
\text { current field study }\end{array}$ \\
\hline $\begin{array}{l}\text { Cichorium intybus L., } \\
\text { Asteraceae }\end{array}$ & Cicoria & Leaves & $\begin{array}{l}\text { HER: depurative } \\
\text { (decoction) }\end{array}$ & & \\
\hline $\begin{array}{l}\text { Crataegus monogyna Jacq., } \\
\text { Rosaceae, } \\
\text { UNISGSANG15012 }\end{array}$ & Bosu & $\begin{array}{l}\text { Leaves and } \\
\text { flowers }\end{array}$ & & $\begin{array}{l}\text { HER: tranquilizing, } \\
\text { hypotensive (tea) }\end{array}$ & Rare \\
\hline $\begin{array}{l}\text { Cynodon dactylon (L.) Pers., } \\
\text { Poaceae, } \\
\text { UNISGSANG18037, } \\
\text { UNISGSANG15013 }\end{array}$ & Gramon, Grmon & Whole plant & $\begin{array}{l}\text { HER: diuretic (tea, often } \\
\text { mixed with corn stigmas } \\
\text { and bearberry leaves) }\end{array}$ & HER: diuretic (tea) & Fairly common \\
\hline $\begin{array}{l}\text { Daphne mezereum L., } \\
\text { Thymelaeceae }\end{array}$ & & Roots & VET: purgative (tea) & & \\
\hline $\begin{array}{l}\text { Eleagnus rhamnoides (L.) } \\
\text { A.Nelson }\end{array}$ & & Fruits & $\begin{array}{l}\text { HER: astringent and anti- } \\
\text { hemorrhagic (crushed } \\
\text { fruits, consumed or topic- } \\
\text { ally applied) }\end{array}$ & & \\
\hline $\begin{array}{l}\text { Epilobium angustifolium L., } \\
\text { Onagraceae, } \\
\text { UNISGSANG15014 }\end{array}$ & Epilobio & Leaves & & HER: anti-cancer & Rare \\
\hline $\begin{array}{l}\text { Equisetum arvense L., } \\
\text { Equisetaceae, } \\
\text { UNISGSANG18015 }\end{array}$ & $\begin{array}{l}\text { Coa d'aval } \\
\text { Erba cavaligna } \\
\text { Erba cavallina }\end{array}$ & Aerial parts & $\begin{array}{l}\text { HER: diuretic, improving } \\
\text { blood circulation } \\
\text { (decoction) }\end{array}$ & $\begin{array}{l}\text { HER: diuretic, improving } \\
\text { joints }\end{array}$ & Rare \\
\hline $\begin{array}{l}\text { Erica arborea L., Ericaceae, } \\
\text { UNISGSANG15016 }\end{array}$ & Erica & Flowering tops & & HER: diuretic (tea) & Rare \\
\hline $\begin{array}{l}\text { Filipendula ulmaria (L.) } \\
\text { Maxim., Rosaceae }\end{array}$ & & Flowering tops & HER: diuretic (tea) & & \\
\hline $\begin{array}{l}\text { Fistulina hepatica (Schaeff.) } \\
\text { With., Fistulinaceae }\end{array}$ & Lenghe & Fruiting body & & FOO: salads & Fairly common \\
\hline $\begin{array}{l}\text { Fragaria vesca } \mathrm{L} \text {., Rosaceae, } \\
\text { UNISGSANG18047 }\end{array}$ & Frola & Fruits & & $\begin{array}{l}\text { FOO: snack; FOO/HER: for } \\
\text { treating intestinal } \\
\text { discomforts (consumed) }\end{array}$ & $\begin{array}{l}\text { Very common (FOO); rare } \\
\text { (FOO/HER) }\end{array}$ \\
\hline $\begin{array}{l}\text { Fumaria officinalis L., } \\
\text { Papaveraceae }\end{array}$ & Fumaria & Leaves & & $\begin{array}{l}\text { HER: for treating eye } \\
\text { inflammations, decoction } \\
\text { (topically applied) }\end{array}$ & Rare \\
\hline $\begin{array}{l}\text { Gentiana acaulis L. and G. } \\
\text { verna L., Gentianaceae, } \\
\text { UNISGSANG18025 and } \\
\text { UNISGSANG15018, } \\
\text { respectively }\end{array}$ & $\begin{array}{l}\text { Cücüc, } \\
\text { Giansanela }\end{array}$ & $\begin{array}{l}\text { Roots and } \\
\text { whole plant ( } G \text {. } \\
\text { acaulis);Flower } \\
\text { buds (G. verna) }\end{array}$ & $\begin{array}{l}\text { FOO/HER: aromatized } \\
\text { wines made from the roots } \\
\text { of G. acaulis (considered } \\
\text { digestive) }\end{array}$ & $\begin{array}{l}\text { FOO/HER: aromatized wine } \\
\text { and liqueurs made from } \\
\text { the whole plant of } G \text {. } \\
\text { acaulis or the flower buds } \\
\text { of G. verna (considered } \\
\text { appetizing, digestive, and } \\
\text { reconstituent) }\end{array}$ & Fairly common \\
\hline $\begin{array}{l}\text { Gentiana lutea L., } \\
\text { Gentianaceae, } \\
\text { UNISGSANG18027 }\end{array}$ & $\begin{array}{l}\text { Giansana } \\
\text { Giansena }\end{array}$ & Roots & $\begin{array}{l}\text { FOO/HER: aromatized } \\
\text { wines (considered } \\
\text { digestive and hepato- } \\
\text { protector) }\end{array}$ & $\begin{array}{l}\text { FOO/HER: aromatized } \\
\text { (white) wines, sometimes } \\
\text { adding lemon peels } \\
\text { (considered appetizing, } \\
\text { digestive, and } \\
\text { reconstituent); VET: } \\
\text { appetizing and digestive } \\
\text { (cows) }\end{array}$ & Very common \\
\hline $\begin{array}{l}\text { Geranium robertianum L., } \\
\text { Geraniaceae, } \\
\text { UNISGSANG15019 }\end{array}$ & Erba roberta & Flowers & & $\begin{array}{l}\text { HER: to treat menstrual } \\
\text { pains (decoction) }\end{array}$ & Rare \\
\hline $\begin{array}{l}\text { Grifola frondosa (Dicks.) } \\
\text { Gray, Grifolaceae }\end{array}$ & Mutun & Fruiting body & & FOO: pickled in oil & Fairly common \\
\hline Hedera helix L., Araliaceae & Edera & Leaves & $\begin{array}{l}\text { HER: wounds (often with } \\
\text { St. John's Wort oleolite) }\end{array}$ & $\begin{array}{l}\text { HER: for treating cradle } \\
\text { cap (tea, externally } \\
\text { applied) }\end{array}$ & Rare \\
\hline
\end{tabular}


Table 1 Local wild food and medicinal plants gathered and used in the study area (Continued)

\begin{tabular}{|c|c|c|c|c|c|}
\hline $\begin{array}{l}\text { Gathered botanical taxon/ } \\
\text { taxa, family/-ies and } \\
\text { voucher specimen code(s) }\end{array}$ & $\begin{array}{l}\text { Local plant } \\
\text { name(s) }\end{array}$ & Used parts & $\begin{array}{l}\text { Folk use recorded in } 1976 \\
\text { (preparation) }\end{array}$ & $\begin{array}{l}\text { Folk use recorded in the } \\
\text { current field study } \\
\text { (preparation) }\end{array}$ & $\begin{array}{l}\text { Frequency of use in the } \\
\text { current field study }\end{array}$ \\
\hline $\begin{array}{l}\text { Helminthoteca echioides (L.) } \\
\text { Holub, Asteraceae }\end{array}$ & Patacun, Scagnet & Leaves & & $\begin{array}{l}\text { FOO: soups; VET: fodder } \\
\text { for rabbits }\end{array}$ & Fairly common \\
\hline $\begin{array}{l}\text { Hepatica nobilis Mill., } \\
\text { Ranunculaceae }\end{array}$ & & Leaves & $\begin{array}{l}\text { HER: anti-horsefly bites } \\
\text { (crushed leaves topically } \\
\text { applied) }\end{array}$ & & \\
\hline $\begin{array}{l}\text { Humulus lupulus L., } \\
\text { Cannabaceae, } \\
\text { UNISGSANG15020, } \\
\text { UNISGSANG18012 }\end{array}$ & Luvertin,Lüvertin & Shoots & & FOO: risotto, omelettes & Very common \\
\hline $\begin{array}{l}\text { Hypericum perforatum L., } \\
\text { Hypericaceae, } \\
\text { UNISGSANG15021, } \\
\text { UNISGSANG18027 }\end{array}$ & $\begin{array}{l}\text { Erba d'San Giuan, } \\
\text { Milapertus, } \\
\text { Stafurà, } \\
\text { Trafurera } \\
\text { Tucram }\end{array}$ & $\begin{array}{l}\text { Aerial parts } \\
\text { and flowers }\end{array}$ & $\begin{array}{l}\text { FOO/HER: liqueurs made } \\
\text { from the leaves } \\
\text { (considered digestive and } \\
\text { intestinal spasmolytic); } \\
\text { digestive, hepato-protector, } \\
\text { cholagogue (decoction of } \\
\text { the flowers); for treating all } \\
\text { skin inflammations (includ- } \\
\text { ing burns and wounds; } \\
\text { oleolite, often extracted in } \\
\text { walnut oil and mixed with } \\
\text { ivy leaves, topically ap- } \\
\text { plied); VET: for treating leg } \\
\text { infections in calves, sheep, } \\
\text { and goats (oleolite mixed } \\
\text { with bran topically applied) }\end{array}$ & $\begin{array}{l}\text { FOO/HER: aromatized } \\
\text { wines made from the } \\
\text { whole aerial parts } \\
\text { (considered tranquilizing), } \\
\text { liqueurs made from the } \\
\text { flowers (considered } \\
\text { tranquilizing and anti- } \\
\text { cholesterolemic); HER: anti- } \\
\text { burns and anti-wounds } \\
\text { (oleolite, topically applied); } \\
\text { anti-stomach ache and } \\
\text { anti-cystitis (oleolite, } \\
\text { drunk) }\end{array}$ & Very common \\
\hline $\begin{array}{l}\text { Hyssopus officinalis L., } \\
\text { Lamiaceae }\end{array}$ & Issop & Flowers & HER: bechic (tea) & & \\
\hline $\begin{array}{l}\text { Juglans regia L., } \\
\text { Juglandaceae, } \\
\text { UNISGSANG1828 }\end{array}$ & Nus & $\begin{array}{l}\text { Unripe fruits } \\
\text { and kernels }\end{array}$ & & $\begin{array}{l}\text { FOO/HER: liqueurs made } \\
\text { from unripe fruits } \\
\text { (considered digestive); } \\
\text { sweets, salads with garlic, } \\
\text { vinegar, and salt (kernels) }\end{array}$ & Very common \\
\hline $\begin{array}{l}\text { Juniperus communis L., } \\
\text { Cupressaceae, } \\
\text { UNISGSANG15022 }\end{array}$ & Genevru & $\begin{array}{l}\text { Branches, bark } \\
\text { and galbules }\end{array}$ & & $\begin{array}{l}\text { FOO: roasted meat } \\
\text { seasoning (galbules); } \\
\text { grappa seasoning } \\
\text { (branches); VET: general } \\
\text { sickness (galbules as } \\
\text { fodder for cows and bark } \\
\text { as fodder for rabbits) }\end{array}$ & $\begin{array}{l}\text { Very common (FOO); rare } \\
\text { (VET) }\end{array}$ \\
\hline $\begin{array}{l}\text { Laburnum anagyroides L., } \\
\text { Fabaceae, } \\
\text { UNISGSANG18020 }\end{array}$ & Aburn & $\begin{array}{l}\text { Branches, } \\
\text { leaves and } \\
\text { flowers }\end{array}$ & & $\begin{array}{l}\text { VET: insect repellent (hang } \\
\text { in the hen-house) }\end{array}$ & Rare \\
\hline $\begin{array}{l}\text { Lapsana communis L., } \\
\text { Asteraceae, } \\
\text { UNISGSANG18060 }\end{array}$ & $\begin{array}{l}\text { Burasa russa, } \\
\text { Scina rusa }\end{array}$ & Leaves & & FOO: soups & Rare \\
\hline Larix decidua Mill., Pinaceae & Pin & $\begin{array}{l}\text { Resin and } \\
\text { young shoots }\end{array}$ & $\begin{array}{l}\text { HER: muscular pains and } \\
\text { arthritis (oleolite, externally } \\
\text { applied); bechic } \\
\text { (decoction) }\end{array}$ & & \\
\hline $\begin{array}{l}\text { Laserpitium siler L., } \\
\text { Apiaceae }\end{array}$ & Apia & Leaves & $\begin{array}{l}\text { HER: anti-bruises (crushed } \\
\text { leaves mixed with pork fat } \\
\text { and topically applied) }\end{array}$ & & \\
\hline $\begin{array}{l}\text { Leccinum scabrum (Bull.) } \\
\text { Gray, Boletaceae }\end{array}$ & Crava & & & FOO: sautéed, fried & Fairly common \\
\hline $\begin{array}{l}\text { Malva sylvestris L., } \\
\text { Malvaceae, } \\
\text { UNISGSANG15025, } \\
\text { UNISGSANG18033 }\end{array}$ & $\begin{array}{l}\text { Malva, Marva, } \\
\text { Riondela }\end{array}$ & $\begin{array}{l}\text { Leaves and } \\
\text { flowers }\end{array}$ & $\begin{array}{l}\text { HER: tranquilizing, anti- } \\
\text { neuralgic, bechic (tea) }\end{array}$ & $\begin{array}{l}\text { FOO: soups (young } \\
\text { leaves); HER: digestive, } \\
\text { anti-inflammatory, anti- } \\
\text { cystitis (tea); for treating }\end{array}$ & Very common \\
\hline
\end{tabular}


Table 1 Local wild food and medicinal plants gathered and used in the study area (Continued)

\begin{tabular}{|c|c|c|c|c|c|}
\hline $\begin{array}{l}\text { Gathered botanical taxon/ } \\
\text { taxa, family/-ies and } \\
\text { voucher specimen code(s) }\end{array}$ & $\begin{array}{l}\text { Local plant } \\
\text { name(s) }\end{array}$ & Used parts & $\begin{array}{l}\text { Folk use recorded in } 1976 \\
\text { (preparation) }\end{array}$ & $\begin{array}{l}\text { Folk use recorded in the } \\
\text { current field study } \\
\text { (preparation) }\end{array}$ & $\begin{array}{l}\text { Frequency of use in the } \\
\text { current field study }\end{array}$ \\
\hline & & & & $\begin{array}{l}\text { eye inflammation (anti- } \\
\text { stye; tea, externally } \\
\text { applied) }\end{array}$ & \\
\hline $\begin{array}{l}\text { Matricaria chamomilla L., } \\
\text { Asteraceae }\end{array}$ & Canamia & Flowering tops & $\begin{array}{l}\text { HER: tranquilizing, digestive } \\
\text { (tea) }\end{array}$ & $\begin{array}{l}\text { HER: tranquilizing (tea); for } \\
\text { treating eye and ear } \\
\text { inflammations (oleolite, } \\
\text { externally applied); to treat } \\
\text { abdominal pains (hot } \\
\text { compress applied to the } \\
\text { belly) }\end{array}$ & Very common \\
\hline $\begin{array}{l}\text { Melissa officinalis L., } \\
\text { Lamiaceae, } \\
\text { UNISGSANG15026, } \\
\text { UNISGSANG18029 }\end{array}$ & Limonaria, Mlissa & Leaves & & $\begin{array}{l}\text { FOO: seasoning; HER: } \\
\text { digestive, emmenagogue } \\
\text { (tea) }\end{array}$ & Very common \\
\hline $\begin{array}{l}\text { Mentha spicata L. and } \\
\text { possibly other } \\
\text { Mentha spp., Lamiaceae, } \\
\text { UNISGSANG18030 }\end{array}$ & Menta & Leaves & HER: digestive (tea) & $\begin{array}{l}\text { FOO/HER: liqueurs } \\
\text { (considered digestive); } \\
\text { HER: digestive (tea); VET: } \\
\text { anti-lice in hens }\end{array}$ & $\begin{array}{l}\text { Very common (vet use } \\
\text { rare) }\end{array}$ \\
\hline $\begin{array}{l}\text { Myosotis sylvatica Hoffm. } \\
\text { (Boraginaceae), } \\
\text { UNISGSANG18011 }\end{array}$ & $\begin{array}{l}\text { Non ti scordar di } \\
\text { me }\end{array}$ & Flowers & & $\begin{array}{l}\text { FOO: salads; HER: for } \\
\text { treating eye inflammations } \\
\text { (topically applied) }\end{array}$ & Rare \\
\hline $\begin{array}{l}\text { Nasturtium officinale R. Br., } \\
\text { Brassicaceae, } \\
\text { UNISGSANG18012 }\end{array}$ & Grisun & Aerial parts & & $\begin{array}{l}\text { FOO: salads (considered } \\
\text { blood depurative) }\end{array}$ & Fairly common \\
\hline $\begin{array}{l}\text { Neoboletus luridiformis } \\
\text { (Rostk.) Gelardi, Simonini \& } \\
\text { Vizzini, Boletaceae }\end{array}$ & Fre & Fruiting body & & FOO: cooked & Rare \\
\hline $\begin{array}{l}\text { Oxalis acetosella L., } \\
\text { Oxalidaceae, } \\
\text { UNISSANG15027 }\end{array}$ & Trampis, Trampin & & & FOO: omelettes & Fairly common \\
\hline $\begin{array}{l}\text { Papaver rhoeas L., } \\
\text { Papaveraceae }\end{array}$ & Papaver & Petals & $\begin{array}{l}\text { HER: tranquilizing, febrifuge } \\
\text { (tea) }\end{array}$ & & \\
\hline $\begin{array}{l}\text { Parietaria officinalis } L . \text {., } \\
\text { Urticaceae }\end{array}$ & & Aerialparts & $\begin{array}{l}\text { HER: anti-kidney stones } \\
\text { (decoction) }\end{array}$ & $\begin{array}{l}\text { HER: diuretic, anti-kidney } \\
\text { stones, for treating shin- } \\
\text { gles (decoction) }\end{array}$ & Fairly common \\
\hline $\begin{array}{l}\text { Persicaria bistorta L. Samp., } \\
\text { Polygonaceae, } \\
\text { UNISGSANG18041 }\end{array}$ & Lenghe buine & Leaves & & $\begin{array}{l}\text { FOO: cooked; VET: fodder } \\
\text { for goats }\end{array}$ & Fairly common \\
\hline $\begin{array}{l}\text { Picea abies (L.) H.Karst., } \\
\text { Pinaceae }\end{array}$ & Sapin & $\begin{array}{l}\text { Resin and } \\
\text { young shoots }\end{array}$ & $\begin{array}{l}\text { HER: muscular pains and } \\
\text { arthritis (oleolite, externally } \\
\text { applied); bechic } \\
\text { (decoction) }\end{array}$ & & \\
\hline $\begin{array}{l}\text { Pinguicula grandiflora Lam., } \\
\text { and possibly } \\
\text { other Pinguicola spp., } \\
\text { Lentibulariaceae, } \\
\text { UNISSANG15028 (P. } \\
\text { grandiflora) }\end{array}$ & Viola tajarda & Leaves & $\begin{array}{l}\text { HER: anti-bruises (chopped } \\
\text { leaves mixed with lard and } \\
\text { topically applied) }\end{array}$ & $\begin{array}{l}\text { HER: vulnerary (topically } \\
\text { applied) }\end{array}$ & Rare \\
\hline $\begin{array}{l}\text { Pinus mugo Turra and } \\
\text { possibly other Pinus spp... } \\
\text { Pinaceae, } \\
\text { UNISGSANG15029 (P. } \\
\text { mugo) }\end{array}$ & Pin & $\begin{array}{l}\text { Resin and } \\
\text { young shoots }\end{array}$ & $\begin{array}{l}\text { HER: muscular pains and } \\
\text { arthritis (oleolite, externally } \\
\text { applied); bechic } \\
\text { (decoction) }\end{array}$ & $\begin{array}{l}\text { FOO/HER: syrups, liqueurs } \\
\text { (considered bechic) }\end{array}$ & Rare \\
\hline $\begin{array}{l}\text { Plantago coronopus L., } \\
\text { Plantaginaceae, } \\
\text { UNISGSANG } 15030\end{array}$ & Piota de galign & Leaves & & FOO: soups & Fairly common \\
\hline $\begin{array}{l}\text { Plantago lanceolata L., } \\
\text { Plantaginaceae, }\end{array}$ & $\begin{array}{l}\text { Cüjet, Erba dii } \\
\text { canarin, Urie du }\end{array}$ & Leaves & $\begin{array}{l}\text { HER: anti-carbuncles, } \\
\text { (crushed leaves, topically }\end{array}$ & $\begin{array}{l}\text { FOO: soups; HER: anti- } \\
\text { carbuncles, anti- }\end{array}$ & Fairly common \\
\hline
\end{tabular}


Table 1 Local wild food and medicinal plants gathered and used in the study area (Continued)

\begin{tabular}{|c|c|c|c|c|c|}
\hline $\begin{array}{l}\text { Gathered botanical taxon/ } \\
\text { taxa, family/-ies and } \\
\text { voucher specimen code(s) }\end{array}$ & $\begin{array}{l}\text { Local plant } \\
\text { name(s) }\end{array}$ & Used parts & $\begin{array}{l}\text { Folk use recorded in } 1976 \\
\text { (preparation) }\end{array}$ & $\begin{array}{l}\text { Folk use recorded in the } \\
\text { current field study } \\
\text { (preparation) }\end{array}$ & $\begin{array}{l}\text { Frequency of use in the } \\
\text { current field study }\end{array}$ \\
\hline $\begin{array}{l}\text { UNISGSANG15031, } \\
\text { UNISGSANG18036 }\end{array}$ & giari & & applied) & $\begin{array}{l}\text { haemorrhoids, (compress, } \\
\text { externally applied) }\end{array}$ & \\
\hline $\begin{array}{l}\text { Plantago major } \mathrm{L} ., \\
\text { Plantaginaceae, } \\
\text { UNISGSANG15037 }\end{array}$ & Piantagn & Leaves & & $\begin{array}{l}\text { FOO: soups; HER: diuretic } \\
\text { (leaves) }\end{array}$ & Rare \\
\hline $\begin{array}{l}\text { Polygonatum multiflorum } \\
\text { (L.) All., Asparagaceae }\end{array}$ & & Rhizomes & $\begin{array}{l}\text { HER: anti-carbuncles (exter- } \\
\text { nally applied) }\end{array}$ & & \\
\hline $\begin{array}{l}\text { Polypodium vulgare L., } \\
\text { Polypodiaceae, } \\
\text { UNISGSANG15032, } \\
\text { UNISGSANG18043 }\end{array}$ & Argalisia & Rhizomes & & FOO: snack & Rare \\
\hline $\begin{array}{l}\text { Potentilla anserina L., } \\
\text { Rosaceae, } \\
\text { UNISGSANG15033 }\end{array}$ & Erb del set virtü & Rhizomes & & $\begin{array}{l}\text { HER: anti-diarrheic, febri- } \\
\text { fuge, anti-stomach ache, } \\
\text { bechic (decoction) }\end{array}$ & Fairly common \\
\hline $\begin{array}{l}\text { Primula vulgaris Huds. and } \\
\text { possibly P. veris L., } \\
\text { Primulaceae, } \\
\text { UNISGSANG15034, } \\
\text { UNISSANG18044 (P. } \\
\text { vulgaris) }\end{array}$ & $\begin{array}{l}\text { Piumbera, } \\
\text { Ptrine }\end{array}$ & $\begin{array}{l}\text { Flowers and } \\
\text { roots }\end{array}$ & $\begin{array}{l}\text { HER: bechic (decoction of } \\
\text { the roots) }\end{array}$ & FOO: soups (flowers) & Fairly common \\
\hline Prunus avium L., Rosaceae & $\begin{array}{l}\text { Cerese, Cerse } \\
\text { büshas }\end{array}$ & $\begin{array}{l}\text { Fruit peduncles } \\
\text { and fruits }\end{array}$ & $\begin{array}{l}\text { HER: febrifuge (tea of the } \\
\text { peduncles) }\end{array}$ & $\begin{array}{l}\text { FOO: snack, jams, } \\
\text { preserved (fruits); HER: } \\
\text { anti-inflammatory, diuretic } \\
\text { (decoction of the } \\
\text { peduncles) }\end{array}$ & Very common \\
\hline Prunus cerasus L., Rosaceae & Griutera & Fruits & & FOO: snack & Very common \\
\hline $\begin{array}{l}\text { Prunus domestica L., } \\
\text { Rosaceae }\end{array}$ & Bergna & Kernels & $\begin{array}{l}\text { HER: vermifuge (crushed } \\
\text { kernels mixed with honey } \\
\text { and consumed) }\end{array}$ & & \\
\hline Prunus padus L., Rosaceae & & Bark & HER: febrifuge (tea) & & \\
\hline $\begin{array}{l}\text { Pulmonaria officinalis } L \text {., } \\
\text { Boraginaceae }\end{array}$ & & Leaves & HER: bechic (tea) & & \\
\hline $\begin{array}{l}\text { Quercus petraea (Matt.) } \\
\text { Liebl. and possibly Q. robur } \\
\text { L., Fagaceae, } \\
\text { UNISGSANG18024 (Q. } \\
\text { petraea) }\end{array}$ & Rol, Rul & $\begin{array}{l}\text { Leaves, bark } \\
\text { and acorns }\end{array}$ & $\begin{array}{l}\text { VET: astringent (decoction } \\
\text { of bark and acorns for } \\
\text { calves) }\end{array}$ & $\begin{array}{l}\text { VET: galactagogue (dried } \\
\text { leaves as fodder for goats) }\end{array}$ & Rare \\
\hline $\begin{array}{l}\text { Ranunculus acris L. } \\
\text { Ranunculaceae, } \\
\text { UNISGSANG15035 }\end{array}$ & Ranuncolo & Flowers & & $\begin{array}{l}\text { HER: rubefacient (applied } \\
\text { under the feet to artificially } \\
\text { generate a rash for } \\
\text { avoiding compulsory } \\
\text { military service) }\end{array}$ & $\begin{array}{l}\text { N/A (remembered from } \\
\text { the past) }\end{array}$ \\
\hline $\begin{array}{l}\text { Rhododendron hirsitum } \\
\text { L. and possibly } R \text {. } \\
\text { ferrugineum, Ericaceae, } \\
\text { UNISGSANG15036 (R. } \\
\text { hirsitum) }\end{array}$ & Brunsai & Flowers & $\begin{array}{l}\text { HER: bechic (decoction); } \\
\text { anti-kidney stones (tea) }\end{array}$ & HER: diuretic (decoction) & Rare \\
\hline $\begin{array}{l}\text { Robinia pseudoacacia } \\
\text { L.Fabaceae, } \\
\text { UNISGSANG18021 }\end{array}$ & Gasia & Inflorescences & & FOO: deep fried & Fairly common \\
\hline $\begin{array}{l}\text { Rosa canina L., Rosaceae, } \\
\text { UNISGSANG18050 }\end{array}$ & Gratacul, Gratacü & Pseudo-fruits & HER: astringent (tea) & $\begin{array}{l}\text { FOO: jams, liqueurs;HER: } \\
\text { diuretic (decoction) }\end{array}$ & Fairly common \\
\hline $\begin{array}{l}\text { Rubus idaeus L., Rosaceae, } \\
\text { UNISGSANG18051 }\end{array}$ & Àmpula & Fruits & & FOO: snack, jams, liqueurs & Very common \\
\hline $\begin{array}{l}\text { Rubus ulmifolius Schott., } \\
\text { Rosaceae, UNISSANG15037, } \\
\text { UNISGSANG18052 }\end{array}$ & Runza & $\begin{array}{l}\text { Young shoots, } \\
\text { unripe and ripe } \\
\text { fruits }\end{array}$ & & $\begin{array}{l}\text { FOO: jams (fruits);soups } \\
\text { (young shoots); } \\
\text { HER: anti-hoarseness }\end{array}$ & Fairly common \\
\hline
\end{tabular}


Table 1 Local wild food and medicinal plants gathered and used in the study area (Continued)

\begin{tabular}{|c|c|c|c|c|c|}
\hline $\begin{array}{l}\text { Gathered botanical taxon/ } \\
\text { taxa, family/-ies and } \\
\text { voucher specimen code(s) }\end{array}$ & $\begin{array}{l}\text { Local plant } \\
\text { name(s) }\end{array}$ & Used parts & $\begin{array}{l}\text { Folk use recorded in } 1976 \\
\text { (preparation) }\end{array}$ & $\begin{array}{l}\text { Folk use recorded in the } \\
\text { current field study } \\
\text { (preparation) }\end{array}$ & $\begin{array}{l}\text { Frequency of use in the } \\
\text { current field study }\end{array}$ \\
\hline & & & & $\begin{array}{l}\text { (gargles of a decoction of } \\
\text { the unripe fruits); anti-diar- } \\
\text { rheic (decoction of the } \\
\text { young shoots); vulnerary } \\
\text { and anti-septic (alcoholic } \\
\text { macerate of the young } \\
\text { shoots, externally applied) }\end{array}$ & \\
\hline $\begin{array}{l}\text { Rumex acetosa L., } \\
\text { Polygonaceae, } \\
\text { UNISGSANG15038, } \\
\text { UNISGSANG18042 }\end{array}$ & Esileu, Isiule & Leaves & & $\begin{array}{l}\text { FOO: salads, omelettes, } \\
\text { sauce (mixed with milk } \\
\text { and ghees) for } \\
\text { accompanying polenta; } \\
\text { HER: diuretic (tea) }\end{array}$ & Fairly common \\
\hline $\begin{array}{l}\text { Rumex alpinus L., } \\
\text { Polygonaceae, } \\
\text { UNISGSANG15039 }\end{array}$ & $\begin{array}{l}\text { Ariei, Lapas, } \\
\text { Rebarbar } \\
\text { d'montagna }\end{array}$ & $\begin{array}{l}\text { Leaves, } \\
\text { inflorescences } \\
\text { and roots }\end{array}$ & $\begin{array}{l}\text { HER: digestive (tea of the } \\
\text { leaves and inflorescences) }\end{array}$ & $\begin{array}{l}\text { FOO: soups,wrapping for } \\
\text { butter (leaves); HER: } \\
\text { digestive (root decoction) }\end{array}$ & $\begin{array}{l}\text { Fairly common (FOO; } \\
\text { wrapping for butter } \\
\text { remembered from the } \\
\text { past); rare (HER) }\end{array}$ \\
\hline $\begin{array}{l}\text { Ruta graveolens L., } \\
\text { Rutaceae }\end{array}$ & Ruta & Leaves & & FOO: grappa seasoning & Fairly common \\
\hline Salix caprea L., Salicaceae & Sales & $\begin{array}{l}\text { Leaves and } \\
\text { bark }\end{array}$ & $\begin{array}{l}\text { HER: anti-callus (crushed, } \\
\text { externally applied) }\end{array}$ & & \\
\hline $\begin{array}{l}\text { Salvia officinalis L., } \\
\text { Lamiaceae, } \\
\text { UNISGSANG18031 }\end{array}$ & Sarvia & Leaves & $\begin{array}{l}\text { HER: digestive (decoction } \\
\text { and liqueur) }\end{array}$ & $\begin{array}{l}\text { HER: tranquilizing, } \\
\text { digestive, reconstituent } \\
\text { (decoction, not to be used } \\
\text { during pregnancy); anti- } \\
\text { septic (washes) }\end{array}$ & $\begin{array}{l}\text { Very common(old local } \\
\text { saying: "Se la fumna a } \\
\text { savèisa la virtü d'la salvia, } \\
\text { a saria mai malavia" = "If } \\
\text { a woman knew the } \\
\text { virtues of sage, she } \\
\text { would never be ill") }\end{array}$ \\
\hline $\begin{array}{l}\text { Sambucus nigra L. and S. } \\
\text { racemosa L., Adoxaceae, } \\
\text { UNISSANG15040 (S. nigra) }\end{array}$ & Sambur & $\begin{array}{l}\text { Young } \\
\text { branches, } \\
\text { flowers and } \\
\text { fruits }\end{array}$ & $\begin{array}{l}\text { FOO/HER: aromatized } \\
\text { wines made from the } \\
\text { flowers and fruits } \\
\text { (considered depurative); } \\
\text { HER: anti-rheumatic (oleo- } \\
\text { lite of the flowers, exter- } \\
\text { nally applied) }\end{array}$ & $\begin{array}{l}\text { FOO: jams (fruits or } \\
\text { flowers), soups (flowers); } \\
\text { HER: anti-herpes, anti- } \\
\text { burns, for treating skin in- } \\
\text { flammations, anti- } \\
\text { haemorrhoids (poultice } \\
\text { mixing bee wax and } \\
\text { young branches); anti- } \\
\text { toothache (flowers, exter- } \\
\text { nally applied); diuretic and } \\
\text { febrifuge (decoction of the } \\
\text { flowers); anti-toothache } \\
\text { and anti-rheumatic (decoc- } \\
\text { tion of the flowers in milk, } \\
\text { externally applied) }\end{array}$ & Very common \\
\hline $\begin{array}{l}\text { Scabiosa columbaria L., } \\
\text { Caryophyllaceae, } \\
\text { UNISGSANG15056, } \\
\text { UNISGSANAG18059 }\end{array}$ & $\begin{array}{l}\text { Gialina grasa, } \\
\text { Gialina grasa }\end{array}$ & Whorls & & FOO: soups & Rare \\
\hline $\begin{array}{l}\text { Sedum telephium L., } \\
\text { Crassulaceae, } \\
\text { UNISSANG15043 }\end{array}$ & Erba dei calli & Aerial parts & & $\begin{array}{l}\text { HER: anti-callus (oleolite, } \\
\text { externally applied) }\end{array}$ & Rare \\
\hline $\begin{array}{l}\text { Sempervivum sp., } \\
\text { Crassulaceae }\end{array}$ & & Leaves & $\begin{array}{l}\text { HER: anti-callus (crushed } \\
\text { leaves topically applied) }\end{array}$ & & \\
\hline $\begin{array}{l}\text { Silene vulgaris (Moench) } \\
\text { Garcke, Caryophyllaceae, } \\
\text { UNISGSANG } 15044\end{array}$ & Ciuchinot, Cöiet & Leaves & & FOO: soup & Fairly common \\
\hline $\begin{array}{l}\text { Silybum marianum (L.) } \\
\text { Gaertn., Asteraceae, } \\
\text { UNISGSANG15045 }\end{array}$ & Cardun & Leaves & & $\begin{array}{l}\text { HER: hepato-protector } \\
\text { (decoction) }\end{array}$ & Rare \\
\hline $\begin{array}{l}\text { Solanum dulcamara L., } \\
\text { Solanaceae }\end{array}$ & Dulcamara & Fruits & & $\begin{array}{l}\text { HER: bechic (decoction } \\
\text { with apples) }\end{array}$ & Rare \\
\hline
\end{tabular}


Table 1 Local wild food and medicinal plants gathered and used in the study area (Continued)

\begin{tabular}{|c|c|c|c|c|c|}
\hline $\begin{array}{l}\text { Gathered botanical taxon/ } \\
\text { taxa, family/-ies and } \\
\text { voucher specimen code(s) }\end{array}$ & $\begin{array}{l}\text { Local plant } \\
\text { name(s) }\end{array}$ & Used parts & $\begin{array}{l}\text { Folk use recorded in } 1976 \\
\text { (preparation) }\end{array}$ & $\begin{array}{l}\text { Folk use recorded in the } \\
\text { current field study } \\
\text { (preparation) }\end{array}$ & $\begin{array}{l}\text { Frequency of use in the } \\
\text { current field study }\end{array}$ \\
\hline $\begin{array}{l}\text { Solanum nigrum L., } \\
\text { Solanaceae, } \\
\text { UNISGSANG15046 }\end{array}$ & Erba morela & $\begin{array}{l}\text { Leaves and } \\
\text { fruits }\end{array}$ & $\begin{array}{l}\text { HER: anti-arthritis and anti- } \\
\text { rheumatic (poultice) }\end{array}$ & $\begin{array}{l}\text { HER: anti-carbuncles (fresh } \\
\text { leaves directly applied) }\end{array}$ & Rare \\
\hline $\begin{array}{l}\text { Stellaria media (L.) Vill., } \\
\text { Caryophyllaceae }\end{array}$ & Purota & Aerial parts & & FOO: salads & Rare \\
\hline $\begin{array}{l}\text { Suillus granulatus (L.) } \\
\text { Rousell, Suillaceae }\end{array}$ & Pinaiolo & Fruiting body & & FOO: cooked & Rare \\
\hline $\begin{array}{l}\text { Tanacetum vulgare L., } \\
\text { Asteraceae }\end{array}$ & T'nea & Leaves & HER: hypotensive (tea) & $\begin{array}{l}\text { VET: digestive and antidote } \\
\text { against Veratrum spp. } \\
\text { ingestion (cows) }\end{array}$ & Rare \\
\hline $\begin{array}{l}\text { Taraxacum officinale (L.) } \\
\text { Weber ex F.H. Wigg, } \\
\text { Asteraceae, } \\
\text { UNISSANG15048 }\end{array}$ & $\begin{array}{l}\text { Cicoria de pra, } \\
\text { Girasui, Taracun }\end{array}$ & $\begin{array}{l}\text { Leaves and } \\
\text { flowers }\end{array}$ & & $\begin{array}{l}\text { FOO: salads, omelettes, } \\
\text { soups;HER: diuretic, liver } \\
\text { protector (tea); bechic } \\
\text { (flower syrup) }\end{array}$ & Very common \\
\hline $\begin{array}{l}\text { Thymus serpyllum L., } \\
\text { Lamiaceae, } \\
\text { UNISGSANG15049, } \\
\text { UNISGSANG18032 }\end{array}$ & Serpolet, Serpul & $\begin{array}{l}\text { Leaves and } \\
\text { flowers }\end{array}$ & $\begin{array}{l}\text { HER: anti-stomatitis } \\
\text { (mouthwash) }\end{array}$ & $\begin{array}{l}\text { FOO: meat, soups, and } \\
\text { mushroom salads } \\
\text { seasoning; smoked with } \\
\text { tobacco; FOO/HER: } \\
\text { aromatized wines } \\
\text { (considered digestive); } \\
\text { HER: digestive and tonic } \\
\text { (tea); flu (external } \\
\text { compress); oral antiseptic } \\
\text { (mouthwash of } \\
\text { decoction); FOO/HER: } \\
\text { liqueurs (considered } \\
\text { digestive); VET: fodder for } \\
\text { rabbits before they are } \\
\text { butchered (for enhancing } \\
\text { flavour) }\end{array}$ & Fairly common \\
\hline $\begin{array}{l}\text { Tilia cordata Mill., } \\
\text { Malvaceae, } \\
\text { UNISGSANG15050 }\end{array}$ & Tij & $\begin{array}{l}\text { Leaves and } \\
\text { flowers (tiöl) }\end{array}$ & $\begin{array}{l}\text { HER: bechic and febrifuge } \\
\text { (tea) }\end{array}$ & HER: bechic (tea) & Very common \\
\hline $\begin{array}{l}\text { Tragopogon pratensis L., } \\
\text { Asteraceae, } \\
\text { UNISGSANG18051 }\end{array}$ & Barbaboch & Leaves & $\begin{array}{l}\text { HER: depurative } \\
\text { (decoction) }\end{array}$ & FOO: boiled, soup & Fairly common \\
\hline $\begin{array}{l}\text { Tussilago farfara L., } \\
\text { Asteraceae }\end{array}$ & Pata d'asu & Leaves & $\begin{array}{l}\text { HER: whooping cough } \\
\text { (fumigations); for treating } \\
\text { insect bites (compress of } \\
\text { the fresh leaves) }\end{array}$ & & \\
\hline $\begin{array}{l}\text { Urtica dioica L., Urticaceae, } \\
\text { UNISGSANG18055 }\end{array}$ & Urtia, Ürtia & $\begin{array}{l}\text { Leaves and } \\
\text { roots }\end{array}$ & $\begin{array}{l}\text { HER: anti-dysmenorrhea } \\
\text { (decoction of the roots) }\end{array}$ & $\begin{array}{l}\text { FOO: soups, risotto, } \\
\text { omelettes, salads (leaves); } \\
\text { HER: cicatrizing, anti- } \\
\text { bruises (external applica- } \\
\text { tions of chopped leaves); } \\
\text { anti-alopecia and hair } \\
\text { strengthening (decoction, } \\
\text { externally applied); VER: } \\
\text { enhancing egg production } \\
\text { (fodder for hens) }\end{array}$ & Very common \\
\hline $\begin{array}{l}\text { Vaccinium myrtillus L., } \\
\text { Ericaceae, } \\
\text { UNISGSANG15057, } \\
\text { UNISSANG18017 }\end{array}$ & Ambrune & Fruits & & $\begin{array}{l}\text { FOO: jams; FOO/HER: } \\
\text { snack ("good for the } \\
\text { eyes"), liqueurs (considered } \\
\text { anti-diarrhoeic); HER: anti- } \\
\text { prostatitis, antiphlogistic of } \\
\text { the urinary tract }\end{array}$ & Very common \\
\hline $\begin{array}{l}\text { Vaccinium vitis-idaea } \mathrm{L} ., \\
\text { Ericaceae, } \\
\text { UNISGSANG18018 }\end{array}$ & Anghertin & Fruits & & FOO: snack & Rare \\
\hline
\end{tabular}


Table 1 Local wild food and medicinal plants gathered and used in the study area (Continued)

\begin{tabular}{|c|c|c|c|c|c|}
\hline $\begin{array}{l}\text { Gathered botanical taxon/ } \\
\text { taxa, family/-ies and } \\
\text { voucher specimen code(s) }\end{array}$ & $\begin{array}{l}\text { Local plant } \\
\text { name(s) }\end{array}$ & Used parts & $\begin{array}{l}\text { Folk use recorded in } 1976 \\
\text { (preparation) }\end{array}$ & $\begin{array}{l}\text { Folk use recorded in the } \\
\text { current field study } \\
\text { (preparation) }\end{array}$ & $\begin{array}{l}\text { Frequency of use in the } \\
\text { current field study }\end{array}$ \\
\hline $\begin{array}{l}\text { Valerianella locusta (L.) } \\
\text { Laterr., Valerianaceae, } \\
\text { UNISGSANG15058, } \\
\text { UNISGSANG18055 }\end{array}$ & $\begin{array}{l}\text { Saladet, Saladet } \\
\text { dle funtene }\end{array}$ & Young leaves & & FOO: salads & Very common \\
\hline $\begin{array}{l}\text { Verbascum thapsus L., } \\
\text { Scrophulariaceae }\end{array}$ & Verbasco & $\begin{array}{l}\text { Leaves and } \\
\text { flowers }\end{array}$ & $\begin{array}{l}\text { HER: bechic (infusion of } \\
\text { the flowers); anti-arthritis } \\
\text { (leaves, externally applied) }\end{array}$ & & \\
\hline $\begin{array}{l}\text { Veronica allionii Vill., } \\
\text { Plantaginaceae }\end{array}$ & Te d'montagna & Leaves & HER: tonic (tea) & & \\
\hline $\begin{array}{l}\text { Veronica chamaedrys } L \text {. } \\
\text { and } V \text {. officinalis } L ., \\
\text { Plantaginaceae }\end{array}$ & & Fiori & HER: febrifuge (tea) & & \\
\hline Viola calcarata L., Violaceae & $\begin{array}{l}\text { Viola, Viuletta } \\
\text { d'muntagna }\end{array}$ & Flowers & HER: bechic (tea) & $\begin{array}{l}\text { HER: bechic, laxative, } \\
\text { intestinal anti- } \\
\text { inflammatory (tea) }\end{array}$ & Fairly common \\
\hline $\begin{array}{l}\text { Viola canina subsp. } \\
\text { montana (L.) Hartm., } \\
\text { Violaceae, } \\
\text { UNISGSANG18057 }\end{array}$ & Viulëtta & Flowers & & $\begin{array}{l}\text { FOO: soup ("müshe morte" } \\
=\text { "dead flies", as the } \\
\text { cooked violets resemble } \\
\text { flies) }\end{array}$ & Rare \\
\hline $\begin{array}{l}\text { Viola odorata L., Violaceae, } \\
\text { UNISGSANG15059 }\end{array}$ & Viola mammola & Flowers & & FOO: soups & Rare \\
\hline Viola tricolor L., Violaceae & Viola d'l pensé & Flowers & $\begin{array}{l}\text { HER: febrifuge, tranquillizer } \\
\text { (tea) }\end{array}$ & & \\
\hline Zea mays L., Poaceae & & Stigma & $\begin{array}{l}\text { HER: diuretic and } \\
\text { antiphlogistic against } \\
\text { urinary tract infections } \\
\text { (tea); }\end{array}$ & & \\
\hline
\end{tabular}

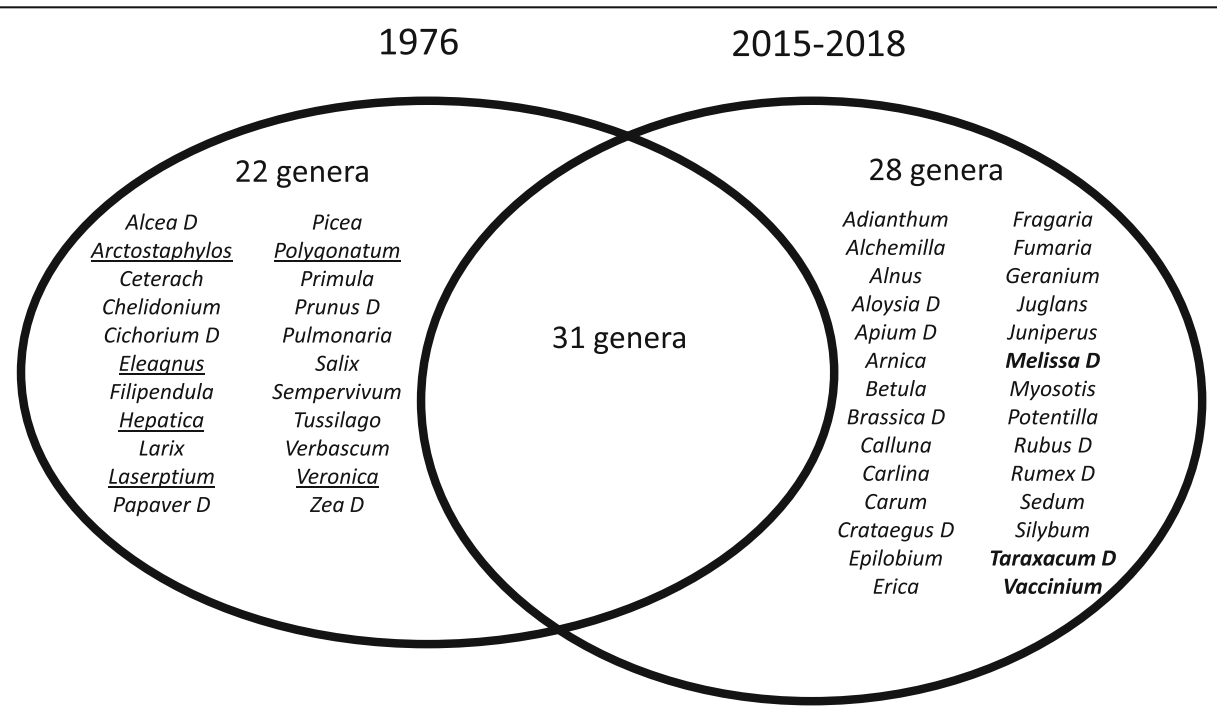

Fig. 2 Venn diagram showing the overlap between the medicinal plant genera gathered in the Seventies and those gathered in 2015-2018 in the Sangone Valley. D: cultivated genera or genera growing in anthropogenic environments; genera mainly growing in forest and higher mountain areas are underlined, while genera very commonly used nowadays are in bold type 
- 68 taxa were recorded in the current study only-as locally gathered plants/mushrooms for food only (33 taxa), for medicinal uses only (23 taxa) or for both (12 taxa);

- 36 taxa have been continuously used during the last 40 years.

Wild food plants and mushrooms still represent an important source of ingredients in the study area today (Table 1). The most important groups of wild food plants are leafy vegetables and mushrooms, which are normally gathered in the spring and autumn, respectively. Most of the recorded wild food and herbal plant reports were previously reported in a few sporadic ethnobotanical studies conducted on the Italian side of the Alps ([60], and references therein [61-65];). Among the traditional and most distinctive recipes, it is worth mentioning the resilience of a soup made with violets called müshe morte ("dead flies"), as the cooked flowers resemble flies.

\section{Cross-temporal comparison}

Figure 2 shows the comparison between the past and current herbal uses of the recorded plant genera. We deemed that considering plant genera and not species was more appropriate given the possible discrepancies in species identification between the datasets and taking into account that under-differentiation does occur for herbaceous plants in the study area (one folk plant name may sometimes correspond to diverse used botanical species within the same genus, see Table 1).

Despite the possible limitations due to the lack of data on the frequency of gathering/use of medicinal plants recorded in the Seventies and the fact that a number of medicinal taxa are not commonly gathered and used nowadays (see Table 1), there was no remarkable quantitative difference between the two medical ethnobotanies (53 recorded herbal genera in the Seventies and 59 in the current study) but qualitatively the difference was substantial.

\section{Discussion}

\section{The renegotiation of situated LEK}

Overall, the data analysis suggests a profound change of herbal LEK which is linked to the very way people lived, and now live, in the valley.

The data collected in 1976 show botanical knowledge linked to different ecosystems in the valley, as well as knowledge of specific, local species. Wild species traditionally gathered in more remote mountain areas could be linked to the disappearance of pastoralist and forestcentred activities, which were prominent in the study area many decades ago.

Figure 2 clearly shows that the gathering and use of plant genera that, accordingly to the Italian botanical reference flora [38], mainly grow in pastures, forests and higher mountain areas (underlined in the figure) has ceased, while the collection of cultivated medicinal plants or medicinal plants growing in more anthropogenic environments [38] has been newly introduced. This is similar to what has been observed for the patterns of change in wild food plant knowledge in Europe [22, 30].

Thus, the research shows a form of LEK that encompasses the entire complexity of the valley, which is compatible with the specificities of local multifunctional farming. This adaptive strategy [73] involves horticultural activities carried out in fields in the lower parts of the valley, hunting and gathering practiced in the forests and summer dairy farming and milk processing conducted in the high pastures. Moreover, plant knowledge was remarkably focused on medicinal uses of herbs. In a country in which a National Health System was instituted only in 1978 [74], the availability of medical treatments and drugs was limited. In this respect, the use of local plants and remedies was a fundamental resource for families; a resource embedded in and specific to the place.

Forty years later, herbal LEK has been transformed, as has the way the community inhabits the Sangone Valley. Dwelling is the result of the different activities a

Table 2 Population change in the municipalities of the Sangone Valley

\begin{tabular}{|c|c|c|c|c|c|c|}
\hline \multirow[t]{2}{*}{ Municipality } & \multicolumn{3}{|c|}{ Inhabitants } & \multicolumn{3}{|c|}{ Population change in \% } \\
\hline & 1951 & 1971 & 2011 & $1951 \rightarrow 2011$ & $1951 \rightarrow 1971$ & $1971 \rightarrow 2011$ \\
\hline$\overline{\text { Coazze }}$ & 3480 & 2819 & 3084 & -11.38 & -18.99 & 9.40 \\
\hline Valgioie & 631 & 311 & 948 & 50.24 & -50.71 & 204.82 \\
\hline Giaveno & 8835 & 10641 & 16281 & 84.28 & 20.44 & 53.00 \\
\hline Trana & 1610 & 1792 & 3881 & 141.06 & 11.30 & 116.57 \\
\hline Reano & 808 & 832 & 1689 & 109.03 & 2.97 & 103.00 \\
\hline Sangano & 549 & 1367 & 3807 & 593.44 & 149.00 & 178.49 \\
\hline Bruino & 786 & 3362 & 8479 & 978.75 & 327.74 & 152.20 \\
\hline Rivalta & 2174 & 10358 & 19245 & 785.23 & 376.45 & 85.80 \\
\hline TOTAL & 18873 & 31482 & 57414 & 204.21 & 66.81 & 82.37 \\
\hline
\end{tabular}

From ISTAT/National Italian Institute of Statistics data collected during the 1951, 1971 and 2011 national census surveys 
community or an individual performs in the landscape and on the basis of this activity they experience and know their surroundings thereby developing their knowledge $[75,76]$. In this respect, the transformation of LEK is the result of a nonlinear history [77] of demography, education and economy.

On the demographic side, while most of the Alpine Valley in the country faced rapid socioeconomic transformation after WWII [52], which led to the abandonment of the valley [78], the Sangone Valley experienced the opposite. Thanks to its position close to Turin and on the main corridor between Italy and France, like neighbouring Val di Susa [47], during the past 40 years, the valley has been repopulated (see Table 2). While from the 1940s to 1960s local inhabitants abandoned the valley, the repopulation of the valley is linked first of all to the opening of a large car manufacturing facility in Rivalta in 1967, as well as other factories in other municipalities in the Sangone Valley [79]. Due to this industrialization, new factory workers, coming from all across Italy [80], have settled in the municipalities of the valley since the 1960s. Moreover, since the 1980s, the valley has been the destination of a new wave of immigrants. In this case, it has been mostly professionals and other people from Turin, who decided to move away from the city looking for better accommodation and environment. Due to the landscape and overall affordability of houses, these newcomers settled in the valley, enriching the cultural diversity of the place. In regard to LEK, these new inhabitants, most of the time, have had little or no knowledge of the valley and its environment but have enriched the culture of the place by bringing with them knowledge concerning different plants from their home communities [81].

This period has not only transformed the demography of the place but also deeply affected the family economy of the valley. As in other parts of the Western Alps [82], it has coincided with a deep transformation of the local economy. In particular, it is linked to a crisis of the multifunctional farming system. Attracted by new and more lucrative jobs in industry or the service sector, locals have abandoned agriculture as the main field of activity. At the same time, the agricultural sector has moved towards industrialization, abandoning traditional practices or marginalizing their role. The same has happened with the role of wild plant and mushroom gathering, which have been relegated to being a marginal hobby with the sole main exception of mushroom harvesting, and in particular boletus mushrooms, which have become a key landmark product for the Sangone Valley, celebrated through advertising and local events, such as the mushroom food festival in Giaveno.

Finally, on the cultural side, as in the rest of the region, the second half of the twentieth century marked a crisis in the transmission of traditional knowledge, as well as environmental knowledge [83]. The crisis was not linked just to the passage from orality to written knowledge, a process that has been vastly explored [11, 84-86], but rather to the expansion and differentiation of (written and oral) sources that compose the mediascape [87] of a local community. In this regard, current environmental knowledge is not the result of a linear intergenerational transmission of knowledge, nor just the result of a process of legitimate peripheral legitimation undergone by the individual within the local community [88]. Rather, it is the assemblage of information gathered by individuals based on their interaction with other members of the community as well as with experts, their formal education and popular literature and also with the information gathered from mass media.

Moreover, "global" remedies emerged as a new phenomenon with three genera (Melissa, Taraxacum, Vaccinium; highlighted in bold type in Fig. 2) that are now widely used but were not even collected forty years ago. In particular, the gathering and use of Alchemilla, Crataegus, Melissa, Silybum, Aloysia, Betula, Fumaria, Taraxacum and Vaccinium spp. may have been spread via popular herbal literature printed during the past four decades in Italy $[66-69,89]$, possibly reinforced first by TV programs and later, during the past decade, by some popular Internet sources and social media [70-72]. In the case of Epilobium angustifolium, a research group has, for example, recently linked the increase in popularity of its herbal use in Eastern European countries to a clear influence of popular literature, likely spread by the work of Austrian herbalist Maria Treben, whose writings have been bestsellers in many countries during the past three decades, and also by a number of Russian internet sources [90].

Undoubtedly, the changes that the valley has experienced have transformed local communities and their knowledge. In light of this change, the transformation in LEK this research has pointed out does not suggest a desituated local environmental knowledge, i.e. depleted and eroded by modernity, as commonly assumed in the literature after the postmodernist turn triggered by the seminal work of Lyotard [91]. Our research, instead, suggests that current herbal LEK is the result of a different way of living in the surroundings that answers to present-day social, economic and cultural needs. As pointed out by Nygren [92], LEK is not antithetical of globalization and modernity, but rather it is embedded in this complexity. The making of this knowledge is complex and comes full of friction [93], through which experiences and knowledge developing inside and outside the locale are assembled. Moreover, the observed co-evolution of LEK reflects the transformation of the local socio-ecological system [7]. Thus, the case of the Sangone Valley shows the plasticity and dynamicity of LEK and outlines a circular relationship between 
environmental change, social change and LEK in which LEK is mutating in response to the transformations of society and the environment. On the other hand, their change also modifies the very way in which the community sees its society and its surroundings.

Despite the major limitation of our study (the lack of data concerning the frequency of gathering/use of the medicinal plants 40 years ago), this circularity suggests that herbal LEK in the study area is not simply eroded or disappearing, but tangible and present, and thus always, but differently, situated. These findings challenge, to some extent, the main conclusions of most ethnobotanical studies that demonstrate or postulate an erosion of medicinal plant knowledge everywhere in the world, especially among the younger generations. This assumption should perhaps be analysed with more caution, also because nature-related knowledge may be affected by the so-called shifting baseline syndrome [94]; that is, fluctuations in several factors (such as the availability of plant resources and plant knowledge that can differ over the course of a lifetime) may cause changes in the reference (baseline) of different generations [94].

Moreover, this finding seems to confirm what Vandebroek and Balick [95] pointed out a few years ago in their pioneering work on the botanical knowledge of Dominican migrants in NYC, in which they showed a statistically measurable increase of knowledge associated with migration and urbanization. The findings are in agreement also with those showing the Estonian evolution of herbal plant knowledge during the past century, during which the use of medicinal plants depending on human influence was remarkably amplified [23].

Ultimately, our data suggest that future directions of research should more carefully look at the adaptive capacity of LEK systems [20], especially in urban environments or sites influenced by urban processes, and avoid the assumptions that some folklore-driven approaches have too hastily proposed by romanticizing "the good old times".

\section{LEK and rural development in the Alps: from museums to participatory planning?}

If the above is true, however, what is the role of LEK in alpine development and the development of the study valley?

In the Sangone Valley, as well as in other areas of the Western Alps, LEK was mostly interpreted as a fragment of intangible heritage; pieces of ancient knowledge preserved only in fragments [96-99]. With this approach, LEK has been placed at the centre of a process of heritagisation and museification, culminating in the development of ethnographic museums as well as specific tourism and commercial communication campaigns $[56,100]$. In this sense, LEK has been turned into an ethno-commodity [101] aimed at addressing cultural and economic issues related to tourism and food business development. However, as pointed out by Comaroff and Comoraff [102], such an approach, even if rewarding in the short term, furthers the cultural and economic dependence of the local community on the outside because it triggers a vicious circle of cultural production in which local communities promote and inflate traditional knowledge in order to suit the needs of tourists and the market. Thus, despite the lexicon and premise, this approach to LEK risks not fostering a real opportunity for sustainable development.

Moving away from this approach by recognizing the importance of LEK as an expression of the actual and current relationship between community and environment, LEK becomes an essential tool for the interpretation and planning of the alpine landscape. In a context in which dynamic, decentralised, participatory planning is recognised as a key tool for sustainable development [102], LEK and its diachronic analysis become a tool for interpreting the transformation a region is undergoing. In particular, LEK can contribute to understanding the movement of the anthropogenic frontier, and therefore the abandonment or intensification of the use of areas in the territory. In our research, this is the case, for example, for the forest and the higher areas of the Sangone Valley. Local ecological knowledge can also help to interpret transformation in the use of the landscape that is not linked directly to economic dynamics, but rather to cultural change. In our research, this is the case for the impact of new popular literature and media in shaping local botanical knowledge, expanding proficiency with exotic plants in front of the loss of knowledge of native species. Lastly, LEK can contribute to developing targeted initiatives in environmental conservation and rural development programs.

Overall, this approach supports the design of better initiatives in the field of rural development, fully embracing the challenge presented by the social change the entire Alps is facing.

\section{Conclusions}

This paper has focused on the transformation of LEK in the Sangone Valley, using folk medico-botanical knowledge as a proxy. The analysis pointed out that the change that botanical knowledge and practices concerning medicinal plants have undergone are mainly represented by ceasing collection and use of a few plants growing in higher mountain environments and by the introduction of new customs linked to species growing in anthropogenic milieus or spread by popular herbal sources. These changes are possibly linked to the social, cultural and economic transformations the communities of the valley have faced over the past 40 years. In light of this complex interconnection, the presented data suggest that LEK has not been eroded, but re-arranged. 
Moreover, these changes, even though profound, have not de-situated LEK from the valley, but rather have defined a new configuration in the relationship between communities and their environmental surroundings. In this respect, we argue that the present configuration of LEK should not be considered a corrupted or fragmentary form of a romanticized ancient and pristine condition. The complex connections between changes in LEK systems and the social, cultural and economic transformations that the valley experienced in the past few decades further suggest that the value of local botanical knowledge does not merely lie in its heritagisation, but rather in its germane implementation as a tool for fostering resilient strategies of sustainable development.

\section{Acknowledgements}

Special thanks are due to all informants who generously shared their local plant knowledge, and to students Maria Ambrosetti Maria, Beatrice Guzzi, Monica Nabaasa, Cristiana Pedrazzini Cristiana, Philipp Oggiano, Maximilian Henkel, Gherardo Marazzani, Luca Nicola and especially Paolo Rossino, who gathered some of the data presented in this article.

\section{Authors' contributions}

AP and MFF designed the study, framed the theoretical and methodological settings, collected and analysed the data and addressed the main trajectories of the outcomes and discussion; they drafted the first version of the manuscript, which was later revised and approved by both authors.

\section{Funding}

This study was partially funded by the University of Gastronomic Sciences, Pollenzo, Italy.

\section{Availability of data and materials}

Original dataset of the work published in 1977 is available at the authors' address and in Table 1. Datasets of the work conducted in 2015 and 2018 are presented in Table 1.

\section{Ethics approval}

Prior informed consent was always verbally obtained in each interview and researchers adhered to the ethical guidelines of the International Society of Ethnobiology [37].

\section{Consent for publication}

N/A.

\section{Competing interests}

The authors declare that they have no competing interest.

Received: 22 April 2020 Accepted: 24 August 2020

Published online: 01 October 2020

\section{References}

1. Quave CL, Pieroni A. A reservoir of ethnobotanical knowledge informs resilient food security and health strategies in the Balkans. Nat Plants. 2015: 14021. https://doi.org/10.1038/nplants.2014.21.

2. Schunko C, Grasser S, Vogl CR. Explaining the resurgent popularity ofthe wild: motivations for wild plant gathering inthe Biosphere Reserve Grosses Walsertal, Austria. J Ethnobiol Ethnomed. 2015;11:55. https:/doi.org/10.1186/s13002-015-0032-4.

3. Reyes-García V, Vila S, Aceituno-Mata L, Calvet-Mir L, Garnatje T, et al. Gendered Homegardens: A Study in Three Mountain Areas of the Iberian Peninsula. Econ Bot. 2010;64:235-47. https://doi.org/10.1007/s12231-010-9124-1.

4. Guadilla-Sáez S, Pardo-de-Santayana M, Reyes-García V. The role of traditional management practices in shaping a diverse habitat mosaic in a mountain region of Norther Spain. Land Use Policy. 2019;89:104235. https:// doi.org/10.1016/j.landusepol.2019.104235.
5. Steinhäuser C. Mountain farmers' intangible values foster agroecological landscapes: case studies from Sierra Santa Victoria in northwest Argentina and the Ladin Dolomites, northern Italy. Agroecol Sust Food. 2020;44:35277. https://doi.org/10.1080/21683565.2019.1624285.

6. Bhatta LD, Udas E, Khan B, Ajmal A, Amir R, et al. Local knowledge based perceptions on climate change and its impacts in the Rakaposhi valley of Gilgit-Baltistan, Pakistan. Int J Clim Chang Str. 2019;12:222-37. https://doi. org/10.1108/IJCCSM-05-2019-0024.

7. Augé M. Oblivion. Minneapolis: University of Minnesota Press; 2004.

8. Ingold T, Kurttila T. Perceiving the Environment in Finnish Lapland. Body Soc. 2000;6:183-96. https://doi.org/10.1177/1357034X00006003010.

9. Lévi-Strauss C. La Pensée Sauvage. Paris: Plon; 1962.

10. Polanyi M. The Tacit Dimension. New York: Doubleday and Co.; 1966.

11. Havelock EA. The Muse Learns to Write. New Haven: Yale University Press; 1986.

12. Warren DM, Slikkerveer LJ, Brokensha D, Dechering WHJC. The Cultural Dimension of Development. London: IT Publication; 1995.

13. Huber T, Pedersen P. Meteorological Knowledge and Environmental Ideas in Traditional and Modern Societies. J Roy Anthropol Inst. 1997; 3: 577-598. https://doi.org/10.2307/303476814.

14. Nazarea VD, editor. Ethnoecology: situated knowledge, located lives. Tucson: University of Arizona Press; 1999.

15. Lauer M, Aswani S. Indigenous Ecological Knowledge as Situated Practices: Understanding Fishers' Knowledge in the Western Solomon Islands. Am Anthropol. 2009;11:317-29. https://doi.org/10.1111/j.1548-1433.2009.01135.x.

16. Whyte KP. On the role of traditional ecological knowledge as a collaborative concept: a philosophical study. Ecol Process. 2013;2:7. https://doi.org/10. 1186/2192-1709-2-7.

17. Linke S, Hadjimichael M, Mackinson S, Holm P. Knowledge for Fisheries Governance: Participation, Integration and Institutional Reform. In: Holm P. Hadjimichael M, Linke S, Mackinson S, editors. Collaborative Research in Fisheries. Cham: Springer; 2020. p. 7-25.

18. Aswani A, Lemahieu A, Sauer WHH. Global trends of local ecological knowledge and future implications. PlosOne. 2018;13:e0195440. https://doi. org/10.1371/journal.pone.0195440.

19. Liu T-M, Chang S-K. Changes in local knowledge and its impacts on ecological resources management: The case of flyingfish culture of the Tao in Taiwan. Mar Policy. 2019;103:74-83. https://doi.org/10.1016/j. marpol.2019.02.031.

20. Duenn P, Salpeteur M, Reyes-García V. Rabari Shepherds and the Mad Tree: The Dynamics of Local Ecological Knowledge in the Context of Prosopis juliflora Invasion in Gujarat, India. J Ethnobiol. 2017;37:561-80. https://doi. org/10.2993/0278-0771-37.3.561.

21. Łuczaj Ł, Szymański WM. Wild vascular plants gathered for consumption in the Polish countryside: a review. J Ethnobiol Ethnomed. 2007;3:17. https:// doi.org/10.1186/1746-4269-3-17.

22. Łuczaj $Ł$. Changes in the utilization of wild green vegetables in Poland since the 19th century: a comparison of four ethnobotanical surveys. J Ethnopharmacol. 2010;24:395-404. https://doi.org/10.1016/j.jep.2010.01.038.

23. Sõukand R, Kalle R. Change in medical plant use in Estonian ethnomedicine: a historical comparison between 1888 and 1994. J Ethnopharmacol. 2011; 135:251-60. https://doi.org/10.1016/j.jep.2011.02.030.

24. Sõukand R, Kalle R. Historical ethnobotanical review of wild edible plants of Estonia (1770s-1960s). Acta Soc Bot Pol. 2012;81:271-81. https://doi.org/10. 5586/asbp.2012.033.

25. Łuczaj Ł, Köhler P, Pirożnikow E, Graniszewska M, Pieroni A, Gervasi T. Wild edible plants of Belarus: from Rostafiński's questionnaire of 1883 to the present. J Ethnobiol Ethnomed. 2013;9:21. https://doi.org/10.1186/1746-4269-9-21.

26. Pieroni A, Rexhepi B, Nedelcheva A, Hajdari A, Mustafa B, Kolosova V, Cianfaglione K, Quave CL. One century later: the folk botanical knowledge of the last remaining Albanians of the upper Reka Valley, Mount Korab, Western Macedonia. J Ethnobiol Ethnomed. 2013;9:22. https://doi.org/10. 1186/1746-4269-9-22.

27. Quave CL, Saitta A. Forty-five years later: The shifting dynamic of traditional ecological knowledge on Pantelleria Island, Italy. Econ Bot. 2016;70:380-93. https://doi.org/10.1007/s12231-016-9363-x.

28. Bussmann RW, Paniagua Zambrana NY, Hart RE, Moya Huanca AL, Ortiz Soria G, et al. Research methods leading to a perception of knowledge loss - one century of plant use documentation among the Chácobo in Bolivia. Econ Bot. 2018;72:81-93. https://doi.org/10.1007/s12231-018-9401-y.

29. Bussmann RW, Paniagua-Zambrana NY, Wood N, Ole Njapit S, Ole Njapit JN, et al. Knowledge loss and change between 2002 and 2017 - a revisit of 
plant-use of the Maasai of Sekenani Valley, Maasai Mara, Kenya. Econ Bot. 2018;72:207-16. https://doi.org/10.1007/s12231-018-9411-9.

30. Łuczaj Ł, Pieroni A, Tardío J, Pardo-de-Santayana M, Sõukand R, et al. Wild food plant use in 21st century Europe: the disappearance of old traditions and the search for new cuisines involving wild edibles. Acta Soc Bot Pol. 2012;81:359-70. https://doi.org/10.5586/asbp.2012.031.

31. Schunko C, Vogl CR. Organic farmers use of wild food plants and fungi in a hilly area in Styria (Austria). J Ethnobiol Ethnomed. 2010;6:17. https://doi.org/ 10.1186/1746-4269-6-17.

32. Chevalier A, Marinova E, Peña-Chocarro E, editors. Plants and People: Choices and Diversity Through Time. Oxford: Oxbow; 2014.

33. Cucinotta F, Pieroni A. "If you want to get married, you have to collect virdura": the vanishing custom of gathering and cooking wild food plants on Vulcano, Aeolian Islands, Sicily. Food Cult Soc. 2018;31:539-67. https:// doi.org/10.1080/15528014.2018.1481263.

34. Lomagno P, Lomagno CR. La fitoterapia popolare della Valle del Sangone. Bollettino della Società Italiana di Farmacia Ospedaliera. 1977;23:445-542.

35. Svanberg I, Łuczaj Ł, Pardo-de-Santayana P, Pieroni A. History and Current Trends of Ethnobiological Research in Europe. In: Anderson EN, Pearsall D, Hunn E, Turner N, editors. Ethnobiology. Oxford: Wiley-Blackwell; 2011. p. 191-214.

36. Pieroni A, Quave CL. Pioneering ethnobotanists in Italy. Pioneering Ethnobotanists in Italy. Early studies on folk plant uses in Piedmont (18801960). In: Svanberg I, Łuczaj Ł, editors. Pioneers in European Ethnobiology. Uppsala: Uppsala University; 2014. p. 263-71.

37. ISE/International Society of Ethnobiology. Code of Ethics. 2008. Available from: http://www.ethnobiology.net/what-we-do/core-programs/ise-ethicsprogram/code-of-ethics/ (Accessed 13 Apr 2020).

38. Pignatti S. Flora d'Italia. Bologna: Edagricole; 1982.

39. The Plant List. Version 1.1. 2013 Available from: http://www.theplantlist.org/ (Accessed 13 Apr 2020).

40. Stevens PF. Angiosperm Phylogeny Website. Version 14. 2017. Available from: http://www.mobot.org/MOBOT/research/APweb/ (Accessed 13 Apr 2020).

41. Index Fungorum. 2020. Available from: http://www.indexfungorum.org/ Names/Names.asp (Accessed 13 Apr 2020).

42. Bonato L, Viazzo PP, editors. Antropologia e Beni Culturali nelle Alpi. Alessandria: Edizioni dell'Orso; 2013.

43. Lazzarini G, editor. Le Risorse di un Territorio Montano Marginale. Milan: Franco Angeli; 2013.

44. Grimaldi P. Cibo e Rito. Palermo: Sellerio; 2012

45. Viazzo PP. Upland Communities. Cambridge: Cambridge University Press; 1989.

46. Touring Club Italiano. Piemonte. Milan: Touring Club Italiano; 1930.

47. Aime M. Fuori dal tunnel. Viaggio antropologico nella Val di Susa. Meltemi: Sesto San Giovanni; 2016.

48. Comoli Mandracci V, editor. Piemonte. Bari: Laterza; 1988.

49. Luraghi R. Agricoltura, Industria e Commercio in Piemonte dal 1846 al 1861. Turin: Palazzo Carignano; 1967.

50. Biddau N, editor. Piemonte Industria. Turin: Photo Publisher; 2007.

51. Pastore A. Alpinismo e Storia d'Italia. Bologna: II Mulino; 2003.

52. Bravo GL. Italiani del Nuovo Millennio. Milan: Franco Angeli; 2013.

53. CREA. L'Agricoltura del Piemonte in Cifre. Rome: CREA; 2017.

54. Maggi M, editor. Piemonte Economico Sociale. Turin: IRES; 2016.

55. Buccheri BM, Passerini GG. Turismo Montano Sostenibile. Turin: Fondazione Santagata per l'Economia della Cultura. I Rapporti di Ricerca; 2019.

56. Zago M. II Ruolo delle Comunità Locali e dei Turisti nella Valorizzazione dei Saperi Tradizionali. Futuribili. 2018;23:193-209. https://doi.org/10.13137/ 1971-0720/27274

57. Regis R. Intorno alla Vitalità del Francoprovenzale nell'Italia di Nord-Ovest. In: Dunoyer $\mathrm{C}$, editor. Actes de la Conférence Annuelle sur l'Activité Scientifique du Centre d'Études Francoprovençales regards Croisés sur la Standardisation du Francoprovençal. Aosta: Region Autonome Vallée d'Aoste; 2019. p. 47-62.

58. Hedrick UP. Sturtevant's Edible Plants of the World. New York: Dover; 1919.

59. Facciola S. Cornocupia II. A Source Book of Edible Plants. Vista: Campong; 1998.

60. Guarrera PM. Usi e tradizioni della flora italiana: medicina popolare ed etnobotanica. Rom: Aracne; 2006.

61. Pieroni A, Giusti ME. Alpine ethnobotany in Italy: traditional knowledge of gastronomic and medicinal plants among the Occitans of the upper Varaita valley, Piedmont. J Ethnobiol Ethnomed. 2009;5:32. https://doi.org/10.1186/ 1746-4269-5-32.
62. Vitalini S, Tomè F, Fico G. Traditional uses of medicinal plants in Valvestino (Italy). J Ethnopharmacol. 2009:121:106-16. https://doi.org/10.1016/j.jep.2008.10.005.

63. Vitalini S, Iriti M, Puricelli C, Ciuchi D, Segale A, et al. Traditional knowledge on medicinal and food plants used in Val San Giacomo (Sondrio, Italy) - An alpine ethnobotanical study. J Ethnopharmacol. 2013;2:517-29. https://doi. org/10.1016/j.jep.2012.11.024.

64. Vitalini S, Puricelli C, Mikerezi I, Iriti M. Plants, people and traditions: Ethnobotanical survey in the Lombard Stelvio National Park and neighbouring areas (Central Alps, Italy). J Ethnopharmacol. 2015;173:435-58. https://doi.org/10.1016/j.jep.2015.05.036.

65. Mattalia G, Quave CL, Pieroni A. Traditional uses of wild food and medicinal plants among Brigasc, Kyé, and Provençal communities on the Western Italian Alps. Genet Resour Crop Ev. 2013;60:587-603. https://doi.org/10.1007/ s10722-012-9859-x.

66. Lieutaghi P. II libro delle erbe: le loro proprietà medicinali, il loro uso culinario, dove trovarle, come coltivarle e raccoglierle. Milan: Rizzoli; 1977.

67. Souzzi RM. Dizionario delle erbe medicinali: oltre duecentocinquanta specie vegetali esaminate nei loro effetti curativi e nella loro utilizzazione pratica. Rom: Newton; 1995.

68. Treben M. La salute dalla farmacia del Signore: consigli ed esperienze con le erbe medicinali. Steyr: Ennsthaler; 1982.

69. Rieffel E. Guarire con le erbe / Suor Bernardina. Milan: Mondadori; 1993.

70. Valussi M. Infoerbe. Available from: https://www.infoerbe.it/. (Accessed 13 Apr 2020).

71. Aboca SpA. Conoscere le piante medicinali. 2016. Available from: http:// www.conoscerelepiantemedicinali.it/. (Accessed 13 Apr 2020).

72. Erbe Spontanee Mangerecce. Available from: https://www.facebook.com/ groups/ErbeSpontaneeMangerecce/. (Accessed 13 Apr 2020).

73. Cohen YA. Man in Adaptation. Chicago: Aldine Publishing; 1968.

74. Luzzi S. Salute e Sanità nell'Italia Repubblicana. Rome: Donzelli; 2004.

75. Janowski M, Ingold T, editors. Imagining Landscape. Farnham: Ashgate; 2012.

76. Ingold T. The Perception of the Environment. London: Routledge; 2000.

77. De Landa M. A Thousand Years of Nonlinear History. New York: Zone Books; 1997.

78. De Rossi A, editor. Riabitare I'Italia. Rome: Donzelli; 2018.

79. Tranfaglia N, editor. Storia di Torino. Vol. 9. Gli Anni della Repubblica. Turin: Einaudi; 1999

80. Gianotti L. Gli Operai della Fiat Hanno Cento Anni. Rome: Editori Riuniti; 1999.

81. Porporato D, Fassino G, editors. Fame di Lavoro. Turin: Consiglio Regionale del Piemonte; 2016.

82. Porcellana V, Fassio G, Viazzo PP, Zanini RC. Socio-demographic Changes and Transmission of Tangible and Intangible Resources. Rev Geogr Alp. 2016;104-3:1-18 https://doi.org/10.4000/rga.3338.

83. Zola L, editor. Ambientare. Milan: Franco Angeli; 2017.

84. Goody J. The Logic of Writing and the Organization of Society. Cambridge: Cambridge University Press; 1986.

85. Ong WJ. Orality and Literacy. London: Methuen; 1982.

86. Leroi-Gourhan A. Gesture and Speech. Cambridge: MIT Press; 1993.

87. Appadurai A. Modernity at Large. Minneapolis: University of Minnesota Press; 1996.

88. Lave J, Wenger E. Situated Learning. Cambridge: Cambridge University Press; 1991.

89. Messéqué M. II mio erbario. Milan: Mondadori; 1982.

90. Sõukand R, Stryamets N, Fontefrancesco MF, Pieroni A. The Importance of tolerating interstices. Babushka markets in Ukraine and Eastern Europe and their role in maintaining local food knowledge and diversity. Heliyon. 2020; 6:e03222. https://doi.org/10.1016/j.heliyon.2020.e03222.

91. Lyotard JF. The Postmodern Condition. Manchester: Manchester University Press: 1984.

92. Nygren A. Local Knowledge in the Environment-Development Discourse. Critique Anthropol. 1999;19:267-88. https://doi.org/10.1177/ $0308275 \times 9901900304$.

93. Tsing A. Friction. Princeton: Princeton University Press; 2005.

94. Hanazaki N, Herbst DF, Simionato Marques M, Vandebroek I. Evidence of the Shifting Baseline Syndrome in Ethnobotanical Research. J Ethnobiol Ethnomed. 2013;9:75. https://doi.org/10.1186/1746-4269-9-75.

95. Vandebroek I, Balick MJ. Globalization and Loss of Plant Knowledge: Challenging the Paradigm. Plos One. 2012;7:e37643. https://doi.org/10.1371/ journal.pone.00376432012.

96. Bonato L, editor. Culture di Confine. Savigliano: L'Artistica; 2013.

97. Corrado F, Porcellana V, editors. Alpi e Ricerca. Milan: Franco Angeli; 2010. 
98. Porporato D. Feste e Musei. Turin: Omega Edizioni; 2007.

99. Regis D, editor. Gli Ecomusei nella Provincia di Cuneo. Turin: Celid; 2009.

100. Slow Food. Guida ai Presidi Slow Food. Bra: Slow Food Editore; 2014.

101. Nared J, Bole D, editors. Participatory Research and Planning in Practice. Cham: Springer; 2020.

102. Comaroff JL, Comaroff J. Ethnicity, Inc. Chicago: Chicago University Press; 2009.

\section{Publisher's Note}

Springer Nature remains neutral with regard to jurisdictional claims in published maps and institutional affiliations.

Ready to submit your research? Choose BMC and benefit from:

- fast, convenient online submission

- thorough peer review by experienced researchers in your field

- rapid publication on acceptance

- support for research data, including large and complex data types

- gold Open Access which fosters wider collaboration and increased citations

- maximum visibility for your research: over $100 \mathrm{M}$ website views per year

At BMC, research is always in progress.

Learn more biomedcentral.com/submissions 